\title{
Genetic architecture of a body color cline in Drosophila americana
}

Lisa L. Sramkoski ${ }^{1 *}$, Wesley N. McLaughlin ${ }^{2 *}$, Arielle M. Cooley ${ }^{2}$, David C. Yuan ${ }^{1}$, Alisha John $^{1}$, and Patricia J. Wittkopp ${ }^{1,2}$

${ }^{*}$ These authors contributed equally to this work.

${ }^{1}$ Department of Molecular, Cellular, and Developmental Biology

${ }^{2}$ Department of Ecology and Evolutionary Biology

University of Michigan, Ann Arbor, MI 48109-1048

Keywords: tan, ebony, genetic heterogeneity, evolution, pigmentation, Drosophila novamexicana

Corresponding author:

Patricia J. Wittkopp

1105 North University Avenue

Ann Arbor, Ml 48109-1048

Fax: 734.763 .0544

Email: wittkopp@umich.edu

Running title: Genetics of body color in D. americana 


\section{Abstract}

2 Phenotypic variation within a species is often structured geographically in clines. In

3 Drosophila americana, a longitudinal cline for body color exists within North America

4 that appears to be due to local adaptation. The tan and ebony genes have been

5 hypothesized to contribute to this cline, with alleles of both genes that lighten body

6 color found in D. americana. These alleles are similar in sequence and function to the

7 allele fixed in D. americana's more lightly pigmented sister species, Drosophila novamexicana. To test this hypothesis, we examined the frequency and geographic

9 distribution of $D$. novamexicana-like alleles of tan and ebony in D. americana. Among

10 alleles from over 100 strains of $D$. americana isolated from 21 geographic locations,

11 we failed to identify additional alleles of tan or ebony with as much sequence similarity

12 to $D$. novamexicana as the alleles previously described. However, using genetic

13 analysis of 51 D. americana strains derived from 20 geographic locations, we identified

14 one new allele of ebony and one new allele of tan segregating in $D$. americana that are

15 functionally equivalent to the $D$. novamexicana allele. An additional 5 alleles of tan also

16 showed marginal evidence of functional similarity. Given the rarity of these alleles,

17 however, we conclude that they are unlikely to be driving the pigmentation cline.

18 Indeed, phenotypic distributions of the 51 backcross populations analyzed indicate a

19 more complex genetic architecture, with diversity in the number and effects of loci

20 altering pigmentation observed both within and among populations of $D$. americana.

21 This genetic heterogeneity poses a challenge to association studies and genomic

22 scans for clinal variation, but might be common in natural populations. 


\section{Introduction}

26 A phenotypic cline describes a gradient of trait variation across geographic space

27 (Huxley 1938). Such clinal variation often correlates with latitude, longitude or altitude,

28 which in turn correlate with environmental factors such as temperature, light, and

29 humidity. Clinal trait variation can arise neutrally from reduced gene flow between

30 geographically distant populations, but natural selection favoring adaptation to varying

31 local environments is more often thought to be responsible -- especially when there is

32 ongoing gene flow among populations (Endler 1977). Genetic variation underlying clinal

33 trait variation is frequently sought by searching for matching allele frequency clines, but

34 this strategy is known to produce many false positives (Lotterhos \& Whitlock 2015;

35 François et al. 2016). Incorporating knowledge of gene function can help overcome this

36 limitation by identifying loci most likely to contribute to trait variation (Stinchcombe \&

37 Hoekstra 2007; Fournier-Level et al. 2011; Hancock et al. 2011; Marjoram et al. 2013).

38 Genome scans can also miss loci contributing to clinal trait variation when traits are

39 controlled by many genes: for such polygenic traits, multiple genotypes can often

40 produce the same phenotype (genetic heterogeneity), which complicates expected

41 allelic variation across a cline (Kawecki \& Ebert 2004; Pritchard \& Di Rienzo 2010;

42 Savolainen et al. 2013; Adrion et al. 2015; Haasl \& Payseur 2016). Here, we use a more

43 targeted approach to investigate the genetic basis of clinal trait variation by directly

44 examining the role of two genes known to affect development of a clinally varying,

45 polygenic trait. More specifically, we examine the contributions of divergent tan and

46 ebony alleles to clinal variation of body color in Drosophila americana. 
The genetic basis of pigmentation differences within and between species has been studied extensively within Drosophila (Massey \& Wittkopp 2016), and pigmentation clines for body color have been reported for many species (e.g., David et al. 1985;

51 David \& Capy 1988; Hollocher et al. 2000; Pool \& Aquadro 2007; Wittkopp et al. 2011;

52 Telonis-Scott et al. 2011). Selection pressures driving these pigmentation clines seem

53 to vary among species, with adaptation proposed to be linked to variation in UV

54 radiation, temperature, and/or humidity (David \& Capy 1988; True 2003; Brisson et al.

55 2005; Rajpurohit et al. 2008; Wittkopp \& Beldade 2009; Clusella-Trullas \& Terblanche

56 2011; Parkash et al. 2012; Matute \& Harris 2013; Bastide et al. 2014; Sillero et al. 2014;

57 Rajpurohit \& Schmidt 2019; Davis \& Moyle 2019). In D. americana, which is found in the

58 United States from the Atlantic coast to just east of the Rocky Mountains, pigmentation

59 varies along a longitudinal cline, with the darkest body color seen among the most

60 eastern populations (Wittkopp et al. 2011). This pigmentation cline is observed despite

61 little evidence of population structure in $D$. americana and signatures of extensive gene

62 flow throughout the species range (Schäfer et al. 2006; Morales-Hojas et al. 2008;

63 Fonseca et al. 2013), suggesting it is due to local adaptation (Wittkopp et al. 2011). D.

64 americana's closest living relative, D. novamexicana, is found in the southwestern

65 United States, west of the Rocky Mountains, and has evolved an even lighter body

66 color, consistent with an extension of the D. americana pigmentation cline (Wittkopp et

67 al. 2011). Although D. americana and D. novamexicana show evidence of reproductive

68 isolation (Ahmed-Braimah \& McAllister 2012), these two species are still able to mate

69 and produce fertile offspring in the lab, allowing genetic dissection of their divergent

70 phenotypes. 
72 Pigmentation differences between $D$. americana and $D$. novamexicana have been

73 linked to divergent alleles of two classic pigmentation genes, ebony and tan, with

74 genomic regions containing these two genes explaining $\sim 87 \%$ of the pigmentation

75 difference (Wittkopp et al. 2003; 2009; Cooley et al. 2012). Proteins encoded by ebony

76 and tan are required for pigment synthesis in Drosophila and catalyze opposite

77 directions of a reversible biochemical reaction converting dopamine to N-beta-alanyl

78 dopamine and vice versa (True et al. 2005; Massey \& Wittkopp 2016). For tan,

79 functionally divergent sites have been mapped to the first intron (Wittkopp et al. 2009)

80 and allele-specific expression analysis in $\mathrm{F}_{1}$ hybrids (Wittkopp et al. 2004) suggests that

81 this divergence affects cis-regulation of tan expression (Cooley et al. 2012). Evidence of

82 cis-regulatory divergence between $D$. americana and $D$. novamexicana has also been

83 detected for ebony using allele-specific expression assays (Cooley et al. 2012);

84 however, the specific sites responsible for this divergence have been difficult to localize

85 because ebony is located in a region of the genome inverted between $D$. novamexicana

86 and D. americana (Wittkopp et al. 2009). Recent work using CRISPR/Cas9 genome

87 editing to generate ebony mutants in both $D$. americana and $D$. novamexicana,

88 however, has shown using reciprocal hemizygosity testing that divergent ebony alleles

89 are indeed responsible for pigmentation differences between these two species (Lamb

90 et al. 2020).

92 The contribution of ebony and tan to pigmentation differences between $D$. americana

93 and D. novamexicana suggests that one or both of these genes might also contribute to

94 variable pigmentation within D. americana. Consistent with this possibility, prior work

95 identified a strain of $D$. americana (DN2) with an allele of ebony that shares both 
sequence and function with the D. novamexicana allele (Wittkopp et al. 2009). A

97 different strain of $D$. americana (A01) was found to carry an allele of tan with sequence

98 and function similar to the D. novamexicana allele (Wittkopp et al. 2009). These alleles

99 seem to have arisen prior to speciation (Wittkopp et al. 2009), suggesting that they were

100 segregating in $D$. americana prior to the divergence of $D$. novamexicana. Based on

101 these data, we hypothesized that differences in the frequency of one or both of these $D$.

102 novamexicana-like alleles among $D$. americana populations might contribute to this

103 species' pigmentation cline. Here, we test this hypothesis by searching over 100 strains

104 of $D$. americana for additional alleles of ebony and/or tan that share similar amounts of

105 sequence identity and/or function to the $D$. novamexicana allele. We then test for

106 associations between pigmentation and segregating sites sampled in ebony and tan.

107 Finally, we analyze pigmentation phenotypes of backcross populations between $D$.

108 novamexicana and 51 strains of $D$. americana to determine how the genetic architecture

109 of body color differs among strains. We find that D. novamexicana-like alleles of ebony

110 and tan are unlikely to explain the body color cline in $D$. americana, and that the genetic

111 architecture is more complex than anticipated, with genetic heterogeneity apparently

112 common within populations affected by local adaptation. These observations suggest

113 that genomic scans for variation in allele-frequencies would fail to find loci underlying

114 this phenotypic cline, as has been predicted for clinally varying polygenic traits

115 (Pritchard \& Di Rienzo 2010; Savolainen et al. 2013; Adrion et al. 2015; Haasl \&

116 Payseur 2016).

\section{Materials and Methods}

119 Fly strains used for sequence analysis 
120 A summary of fly strains used for sequence analysis is provided in Supplementary

121 Table 1. The "A01" strain of D. americana (15010-0951.01) and "N14" strain of $D$.

122 novamexicana (15010-1031.14) were obtained from the Drosophila Species Stock

123 Center (Tucson, AZ). The remaining 112 strains of $D$. americana were generously

124 provided by Dr. Bryant McAllister (University of lowa), who collected the progenitors of

125 these isofemale lines from wild populations between 1996 and 2007 at 21 sites sampled

126 within the population range of $D$. americana in the United States. All flies were reared on a

127 diet of standard yeast-glucose media at $20^{\circ} \mathrm{C}$. Please note that we refer to different

128 collection sites as different populations in the main text for simplicity even though

129 patterns of sequence variation show no evidence of population structure in $D$.

130 americana other than for chromosomal fusions and inversions (Schäfer et al. 2006;

131 Morales-Hojas et al. 2008; Wittkopp et al. 2011; Fonseca et al. 2013).

133 DNA sequence analysis

134 We PCR amplified and Sanger sequenced 579 bp of ebony spanning exons 5-8 and

$1351328 \mathrm{bp}$ of tan from intron 1. (Note that we originally targeted the large first intron of

136 ebony, but polymorphisms among strains caused all primer pairs tested to amplify

137 inconsistently among strains.) After removing low quality bases from raw Sanger

138 sequence reads based on Phred scores, we aligned sequences of ebony from 109

139 strains of $D$. americana plus 1 strain of $D$. novamexicana and sequences of tan from

140102 strains of D. americana plus 1 strain of $D$. novamexicana using the ClustalW

141 algorithm (Thompson et al. 1994) in CodonCode Aligner (version 8.0.2,

142 https://www.codoncode.com/); sequence was obtained for both genes from 99 strains of

143 D. americana (Supplementary Table 1). Only a single strain of $D$. novamexicana was 
144 analyzed in this work because prior work has shown very low levels of polymorphism in

145 this species (Orsini et al. 2004; Caletka \& McAllister 2004; Wittkopp et al. 2009).

146 Sequence alignments used for analysis are provided as Supplementary File 1(ebony)

147 and Supplementary File 2 (tan) and were submitted to GenBank with ID numbers

148 MT350927 - MT351036 for ebony and MT350824 - MT350926 for tan.

150 Gene trees and haplotype network analysis

151 Phylogenetic trees inferring evolutionary relationships among the alleles sampled for

152 ebony and tan were produced using the Maximum Likelihood method based on the

153 Tamura-Nei model of nucleotide substitutions (Tamura \& Nei 1993) in MEGA7 (Kumar

154 et al. 2016). A bootstrap consensus tree was inferred from 100 replicates (Felsenstein

155 1985), with branches supported by less than $50 \%$ of the replicates collapsed. As

156 described in MEGA7, trees used to start the heuristic search were generated using the

157 Neighbor-Join and BioNJ algorithms, with pairwise distances estimated using the

158 Maximum Composite Likelihood (MCL) approach. Topologies with superior log

159 likelihood values were then selected as initial trees. Sites for which $5 \%$ of the strains

160 had alignment gaps, missing data, or ambiguous bases were excluded from this

161 analysis. Because linkage disequilibrium is low within D. americana (Wittkopp et al.

162 2009), we also assessed the sequence similarity among alleles using Median Spanning

163 Networks (Bandelt et al. 1999) (as implemented in PopART (www.popart.otago.ac.nz;

164 March 15, 2015 version, downloaded September 12, 2019) with the epsilon parameter 165 set to 0.

167 Fly strains used for genetic analysis 
168 The genetic basis of pigmentation differences between $D$. americana and $D$. novamexicana

169 was examined for 51 of the $D$. americana strains established and provided by Dr. Bryant

170 McAllister (University of lowa) (McAllister et al. 2008; Sheeley \& McAllister 2008). As shown

171 in Supplementary Table 1, these strains of $D$. americana included 5 strains from each of two

172 locations, 4 strains from each of two locations, 3 strains from each of six locations, 2 strains

173 from each of five locations, and 1 strain from each of five locations. The eastern-most

174 location was Killbuck, Ohio (40.711809, -82.005472), the western- and northern-most

175 location was Niobrara, Nebraska (42.74821, -98.051519), and the southern-most

176 collection site was Sneads, Florida (30.708495, -84.910637). Together, these 51 strains

177 came from 20 of the 21 locations from which strains included in the sequence analysis

178 described above were derived (Supplementary Table 1).

180 Fly crosses for genetic analysis

181 Virgin females were isolated from each of the 51 strains of $D$. americana used for

182 genetic analysis and mated with $D$. novamexicana males to create $\mathrm{F}_{1}$ hybrids. From

183 each of these $F_{1}$ hybrid populations, virgin females were again collected and then

184 mated to $D$. novamexicana males. Male flies were collected from the $\left(\mathrm{BC}_{1}\right)$ progeny

185 produced by each backcross within 3 days of eclosion and aged for one week to 186 ensure pigmentation was fully developed. Each of these $\mathrm{BC}_{1}$ males carried an $\mathrm{X}$

187 chromosome and one copy of each autosome that was a unique recombination of

188 alleles from the $D$. novamexicana and $D$. americana strains crossed to generate its $F_{1}$

189 hybrid mother. These different recombinant chromosomes caused pigmentation to vary

190 among $\mathrm{BC}_{1}$ flies from each cross. The $\mathrm{Y}$ chromosome and the other copy of each

191 autosome in the $\mathrm{BC}_{1}$ males was always inherited from the $D$. novamexicana father. 
193 Phenotyping Pigmentation in Backcross Progeny

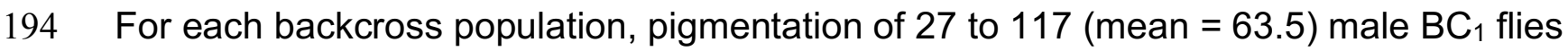

$1957-10$ day old were scored based on the color visible in the dorsal abdominal cuticle of

196 live flies. We found that pigmentation phenotypes did not vary continually in these

197 backcross populations, but rather fell into distinct classes, consistent with prior work

198 (Wittkopp et al. 2003; 2009). The number of distinct pigmentation classes used to

199 score each backcross population was based on the number of distinct pigmentation

200 phenotypes observed: we observed four to eight distinct classes of pigmentation

201 phenotypes in each of the $51 \mathrm{BC}_{1}$ populations. The lightest class was always

202 designated as category " 1 " with increasing class numbers corresponding to

203 progressively darkening pigmentation. For example, in a backcross population with four

204 total pigmentation classes, class "4" would contain the darkest flies, whereas in a

205 backcross population with seven total pigmentation classes, class "4" would contain

206 flies with mid-range pigmentation. The number of pigmentation classes as well as the

207 assignment of individual flies to a particular pigmentation class was determined by

208 independent observations from at least two researchers. These pigmentation

209 phenotype scores are shown for each fly in Supplementary Table 3.

211 DNA Extractions

212 From each of the 51 backcross populations, DNA was extracted from each male $\mathrm{BC}_{1}$ fly

213 using a method similar to that described in Gloor et al. (1993) except that the protocol

214 was scaled for efficient processing of 3238 flies. Briefly, each fly was placed into a well

215 of a 96-well plate (GeneMate\# T3031-21) with a glass bead and 50 $\mu \mathrm{L}$ of a 1:99 
216 Proteinase K/Engel's Buffer solution. Plates were sealed and shaken in a Qiagen

217 Retsch MM301 Tissue Lyser until the glass bead had pulverized the fly in each well.

218 The plates were then incubated at $37^{\circ} \mathrm{C}$ for 30 minutes to allow protein digestion and

219 then incubated at $95^{\circ} \mathrm{C}$ for 2 minutes to inactivate Proteinase K. Extracted DNA was

220 stored at $4^{\circ} \mathrm{C}$ until used for genotyping.

\section{Genotyping}

223 Molecular genotyping assays were used to determine whether each of the $\mathrm{BC}_{1}$ males

224 scored for pigmentation carried the D. americana and/or D. novamexicana alleles of

225 three pigmentation genes: yellow, tan, and ebony. Because yellow and tan are located

226 on the X chromosome, each male carried only one species' allele, either the mother's or

227 the father's allele. By contrast, because ebony is located on an autosome, $\mathrm{BC}_{1}$ males

228 could either be heterozygous for the $D$. americana and $D$. novamexicana alleles or

229 homozygous for the D. novamexicana allele.

230 For yellow and tan, differences in length between PCR products amplified from

231 the $D$. americana and $D$. novamexicana alleles were used to genotype BC1 flies. For

232 tan, a forward primer (5'-CGAGTTTTTATTCCCACTGAATTAT-3') and a reverse primer

233 (5'-GGGTTCGTCTTATCCACGAT-3') were used to amplify a 100bp product for the $D$.

234 americana tan allele and a 64bp product for the D. novamexicana tan allele. For yellow,

235 depending on which $D$. americana strains was used to generate the $\mathrm{BC}_{1}$ males being

236 genotyped, one of two forward primers was used [yellow forward-1 (5'-

237 CCAAAAGGACAACCGAGTTT-3') or yellow forward-2 (5'-

238 CTAAACATGCCTGAAAATCAATCACGGA-3')] with a yellow reverse primer (5'-

239 AGTCGATTGCCAAAGTGCTC-3'). These different forward primers were necessary 
240 because of differences in yellow DNA sequence among the $D$. americana strains. For

241 most backcross populations, the yellow forward-1 primer paired with the yellow reverse

242 primer generated a 349bp product for the $D$. americana yellow allele and a $372 \mathrm{bp}$

243 product for the D. novamexicana yellow allele. The yellow-forward-2 primer was used to

244 analyze $\mathrm{BC}_{1}$ males from the six strains of $D$. americana (IR0436, LR0540, FP9946,

245 DI0562 MK0738, and SC0708) for which the yellow forward-1 primer and yellow reverse

246 primer did not produce any visible differences in length between the $D$. americana and

247 D. novamexicana alleles. For these six strains, genotyping was performed by using the

248 yellow forward-2 primer and the yellow reverse primer to amplify a region of yellow

249 using PCR and then digesting the PCR product with Dral, which cut only the $D$.

250 novamexicana yellow allele. All digested and undigested PCR products were run on $2 \%$

251 agarose gels and visualized using Ethidium Bromide.

252 For ebony, we were unable to identify PCR products that were easily

253 distinguishable for $D$. americana and $D$. novamexicana alleles through either amplicon

254 length or restriction digest. Therefore, we genotyped flies at the ebony locus using

255 pyrosequencing (Ahmadian et al. 2000). The PCR product used for pyrosequencing

256 was generated using the forward primer, 5'-AGCCCGAGGTGGACATCA-3', and the

257 biotinylaed reverse primer, 5'-*GTATGGGTCCCTCGCAGAA-3' ( ${ }^{*}$ notates biotinylation).

258 These PCR products were processed, and pyrosequencing performed, as described in

259 Wittkopp et al. (2008). The pyrosequencing primer used had the sequence 5'-

260 CGAGGTGGACATCAAGT-3'. This pyrosequencing assay for ebony used two single

261 nucleotide differences to differentiate between the $D$. americana and $D$. novamexicana

262 ebony alleles. Specifically, the sequences analyzed by pyrosequencing were 5'-

263 CCAAGCTGCT-3' for the D. americana allele and 5'-CGAAGCTTCT-3' for the $D$. 
264 novamexicana allele, where the bolded letters indicate bases used to discriminate

265 between the two alleles.

266 Genotyping data for yellow, tan, and ebony in the $\mathrm{BC}_{1}$ males is summarized in

267 Supplementary Table 4, where $0=$ hemizygous for the $D$. americana allele for yellow

268 and tan and heterozygous for ebony and $1=$ hemizygous $D$. novamexicana allele for

269 yellow and tan and homozygous for ebony. The 96-well plate containing the DNA

270 sample from each fly is also indicated in Supplementary Table 4.

272 Comparing function of $\mathrm{D}$. americana ebony, tan, and yellow alleles to D. novamexicana

273 To determine whether the $D$. americana allele of yellow, tan, and/or ebony from each of

274 the 51 strains of $D$. americana examined was functionally equivalent to the $D$.

275 novamexicana allele of the same gene, we calculated the difference between the mean

276 pigmentation scores of flies inheriting the $D$. americana or $D$. novamexicana allele from

277 their mother in each backcross population. Statistical significance of this difference was

278 assessed for each gene in each backcross using a null distribution of pigmentation

279 differences generated from 10,000 permuted datasets in which the genotypes of the

280 focal gene were shuffled relative to the pigmentation phenotypes. The null hypothesis

281 tested by these permutations was that the $D$. americana and $D$. novamexicana alleles

282 of the focal gene had indistinguishable effects on pigmentation (i.e., that the two alleles

283 are functionally equivalent). This method of testing for statistical significance directly

284 accounts for the differences in sample sizes and allele frequencies among

285 backcrosses. A correction for multiple testing was performed with the p.adjust function

286 with the method=fdr option, which implements the false discovery rate correction as 
287 described in Benjamini \& Hochberg (1995). These adjusted p-values are reported in 288 Supplementary Table 5.

290 Association testing

291 To test for an association between pigmentation and segregating sites in tan and

292 ebony, we used a more quantitative, continuous measure of pigmentation than the

293 pigmentation classes described for backcross populations above. This pigmentation

294 data came from dataset B in Wittkopp et al. (2011) for strains from the DN, II, MK, NN,

295 OC, SC, and WS populations. For the remaining strains, we generated comparable

296 quantitative measurements of pigmentation using the same protocol as described for

297 dataset B in Wittkopp et al. (2011). Briefly, a custom-built fiber optic probe was used to 298 measure light reflected off the fly's abdominal cuticle, with 5 measurements taken per

299 fly and 6-20 flies analyzed per strain. A WS-1 Diffuse Reflection Standard (Ocean

300 Optics) was used to calibrate the probe for each set of measurements and strains were

301 scored in a random order. To minimize the effects of outlier measurements, the median

302 measure of pigmentation observed for each fly was used for analysis. These medians

303 (Supplementary Table 2) were fitted to a linear model including strain as a fixed effect

304 and replicate fly as a random effect with Imer function in the Ime4 R package, and the

305 least-square means were extracted for each strain using the Ismeans function in the

306 Ismeans R package.

308 Variable sites were then identified in tan and ebony using the same sequence

309 alignments used for phylogenetic analysis (Supplementary Files 1 and 2). Sites with

310 the minor allele present in less than 5 strains as well as sites containing indels were 
311 excluded prior to association testing. Each of the remaining variable sites for $\tan (\mathrm{N}=$

$31274)$ and ebony $(\mathrm{N}=40)$ was then tested for an association with pigmentation by fitting

313 the Ismean estimate of pigmentation for each strain to a general linear model (function

$314 \mathrm{~g} / \mathrm{m}$ in R) containing each of the variable sites as a fixed effect.

Standardizing pigmentation classes among strains

317 One representative male fly from each phenotypic class in each backcross was imaged

318 as a visual reference using a Scion Visicapture 1.2 and Scion Corporation Model CFW-

319 1308C color digital camera. These images were processed using Photoshop CS6

320 (Adobe, San Jose, CA), with a constant color adjustment applied to all photos collected

321 on the same day to control for day-to-day variation in imaging conditions. These

322 adjustments were performed to make the digital images more closely match the fly's

323 appearance under the microscope. The parameters for each day's adjustment were

324 determined based on images of a set of standards consisting of seven dissected

325 abdominal cuticles with a range of pigmentation phenotypes. Photos of these cuticle

326 standards were collected interleaved within each batch of $\mathrm{BC}_{1}$ flies. For comparisons

327 among flies from all 51 backcross populations, we used the representative images from

328 each category in each backcross to convert backcross-specific pigmentation scores to

329 a common 8-category pigmentation scale (Supplementary Table 3). After phenotyping,

330 all flies were stored at $-80^{\circ} \mathrm{C}$.

332 Comparing distributions of backcross phenotypes among strains

333 Correspondence analysis (CA), which is similar to principal components analysis but for

334 categorical response variables, was used to reduce the dimensionality of the 
335 distributions of pigmentation classes from backcross (BC1) populations among strains.

336 This analysis was performed using the CA function in the FactoMineR package (Lê et

337 al., 2008) for $\mathrm{R}$ and visualized using factoextra $\mathrm{R}$ package. We then calculated the

338 Euclidean distance between strains in the Dimension 1 and Dimension 2 space from

339 the CA analysis to compare the similarity in backcross pigmentation distributions for

340 strains that were and were not from the same collection site. Euclidean distances

341 between all pairs of strains were calculated using the distances function in the

342 distances $\mathrm{R}$ package.

344 Statistical analyses

$345 \mathrm{R}$ code used for this work is provided in Supplementary File 3. This code was run in

346 RStudio (Version 1.2.5033) using R version 3.6.2 (2019-12-12).

$348 \quad$ Results

350 Comparing sequence of $\mathrm{D}$. americana ebony and tan alleles to D. novamexicana

351 alleles

353 As described in the Introduction, pigmentation differences between $D$. americana and

354 D. novamexicana (Figure 1A) are primarily due to changes in the ebony and tan

355 genes, which control the balance between dark (black and brown) and light

356 (yellow/tan) pigments (Figure 1B). The DN2 strain of D. americana (from Duncan,

357 Nebraska) and the A01 strain of D. americana (from Poplar, Montana) have been

358 shown to carry alleles of ebony and tan, respectively, similar in sequence and function 
359 to the $D$. novamexicana alleles of these genes (Wittkopp et al. 2009). These

360 observations suggest that differences in the frequency of $D$. novamexicana-like alleles

361 among populations of $D$. americana might underlie the longitudinal cline of body color

362 observed within this species. To test this hypothesis, we examined the frequency and

363 geographic distribution of such alleles first by comparing sequences of ebony and tan

364 from over 100 strains of $D$. americana to orthologous sequences from the N14 strain of

365 D. novamexicana. The $D$. americana strains examined were derived from flies

366 captured at 21 different sites within the United States and included DN2 and A01

367 (Figure 1C, Supplementary Table 1).

369 Phylogenetic trees built from these sequences using the maximum likelihood method

370 implemented in MEGA7 (Kumar et al. 2016) confirmed that the ebony allele from the

371 DN2 strain of $D$. americana is more similar to the $D$. novamexicana allele than to other

372 alleles from D. americana (Figure 1D). We failed to find, however, any additional ebony

373 alleles from the 109 new strains of $D$. americana sampled that clustered as closely with

374 D. novamexicana (Figure 1D). Similarly, phylogenetic trees confirmed that the tan

375 allele from the A01 strain of $D$. americana was the only allele among those sampled

376 from 102 strains of $D$. americana that is more closely related to the $D$. novamexicana

377 allele than to other D. americana alleles (Figure 1E). Analyzing these sequences with

378 Minimum Spanning Networks implemented in PopArt (www.popart.otago.ac.nz) also

379 showed that the DN2 and A01 alleles of ebony and tan, respectively, were most similar

380 to the D. novamexicana allele (Supplementary Figures 1 and 2). Taken together, these

381 data indicate that alleles of ebony and tan with sequences closely related to the $D$. 
382 novamexicana allele are rare within $D$. americana and thus unlikely to explain the

383 pigmentation cline observed.

384

Comparing function of D. americana ebony and tan alleles to D. novamexicana alleles

387 To determine whether other $D$. americana alleles of ebony and/or tan might have

388 functional similarity to $D$. novamexicana alleles despite their greater sequence

389 divergence, we crossed virgin females from 51 strains of $D$. americana derived from 20

390 populations (Supplementary Table 1 ) to $D$. novamexicana, and then backcrossed the

$391 \quad F_{1}$ hybrid females to $D$. novamexicana males (Figure 2A). The backcross (BC1)

392 progeny inherited recombinant maternal chromosomes that contain sequences from

393 both their $D$. americana and $D$. novamexicana parents and paternal chromosomes with

394 only D. novamexicana alleles (Figure 2A). Pigmentation was scored for all male flies in

395 each backcross population $(\mathrm{N}=27$ to 117 , mean $=63.5)$, and then each male was

396 genotyped for ebony, tan, and another pigmentation gene, yellow (Supplementary

397 Table 2). The yellow gene was included as a negative control in this study because

398 prior work has shown that it does not contribute to pigmentation divergence between

399 D. americana and D. novamexicana (Wittkopp et al. 2003; 2009).

400

401 Consistent with prior descriptions of backcross populations between $D$. americana and

402 D. novamexicana (Wittkopp et al. 2003; 2009), body color did not vary continuously

403 within the $\mathrm{BC}_{1}$ populations. Rather, a limited number of distinct pigmentation

404 categories were observed in each cross. The number of pigmentation classes ranged

405 from four to eight among backcross populations produced by different strains; 
examples of pigmentation classes for five strains are shown in Figure 2B. The lightest

407 (most yellow) body color phenotype in each backcross was assigned to category 1,

408 with subsequent category numbers corresponding to progressively darker

409 pigmentation.

411 To test for functional divergence of ebony, tan, or yellow alleles between $D$.

412 novamexicana and each strain of $D$. americana, we calculated the difference in mean

413 pigmentation score between flies that inherited the $D$. americana or $D$. novamexicana

414 allele of each gene from their mother. For each gene and each $\mathrm{BC}_{1}$ population, the

415 statistical significance of the pigmentation difference was determined by comparing it

416 to a distribution of differences observed in 10,000 permuted datasets in which the

417 genotypes were shuffled relative to the phenotypes. A false discovery rate correction

418 for multiple tests (Benjamini \& Hochberg 1995) was then applied, and an adjusted p-

419 value cut-off of 0.05 was used to assess statistical significance. That is, tests with $\mathrm{P}<$

$420 \quad 0.05$ were interpreted as evidence of functionally divergent alleles between $D$.

421 novamexicana and the $D$. americana strain tested, whereas tests with $\mathrm{P} \geq 0.05$ were

422 taken as evidence that the $D$. novamexicana and $D$. americana alleles were

423 functionally equivalent. As expected, yellow alleles of $D$. americana and $D$.

424 novamexicana appeared to be functionally equivalent for all strains tested $(P>0.14$ in

425 all cases; Supplementary Table 5; Supplementary Figure 3), further supporting the

426 observation that yellow does not contribute to pigmentation divergence between these 427 two species. 
429 For ebony, all but one strain of $D$. americana tested showed evidence of functional

430 divergence between $D$. americana and D. novamexicana (Supplementary Table 5;

431 Supplementary Figure 4). This one exception (strain DN0748x37, Figure 2C) had a p-

432 value of 0.18 , suggesting that the ebony allele in this strain is functionally equivalent to

433 the D. novamexicana ebony allele. Like the DN2 strain originally found to carry a $D$.

434 novamexicana-like ebony allele, the DN0748x37 strain was collected from Duncan,

435 Nebraska, but it was collected seven years later than the DN2 strain and did not share

436 as much sequence similarity with the D. novamexicana allele as the DN2 allele (Figure

437 1D, Supplementary Figure 1). These observations suggest that more than one allele of

438 ebony similar to $D$. novamexicana in function is segregating in the Duncan, Nebraska

439 population. This population is located near the western edge of $D$. americana's range

440 (Figure 1C) and has some of the lightest pigmentation observed in D. americana

441 (Wittkopp et al. 2011).

442

443 For tan, one strain of D. americana (DA0626) showed evidence of being functionally

444 equivalent to the $D$. novamexicana allele $(P=0.16$, Figure $2 \mathrm{D}$, Supplementary Table 4).

445 This strain was not any more similar in sequence to the $D$. novamexicana tan allele than

446 other alleles of $D$. americana that showed evidence of functional divergence (Figure 1E,

447 Supplementary Figure 2). Five other D. americana strains showed marginal evidence of

448 being functionally equivalent to the $D$. novamexicana allele $(\mathrm{P}$-values $=0.05$ or 0.06 ,

449 Supplementary Figure 5, Supplementary Table 5). With all other strains showing P-

450 values $<0.0001$ (Supplementary Table 5), these five alleles are interpreted as being at

451 least functionally distinct from the majority of $D$. americana tan alleles, if not equivalent

452 to the $D$. novamexicana tan allele. Two of these five alleles were found in strains 
453 collected from the same population (SC0708, SC0718) near the western edge of the

454 species range; however, the other three alleles (II0710, G9647, FP9918, DA0626) as

455 well as the DA0626 allele were found in strains isolated from populations spread

456 throughout the species range (Figure 1C).

458 The frequency and geographic distribution of ebony and tan alleles similar in function

459 to their D. novamexicana orthologs again suggests that they are unlikely to be primarily

460 responsible for the pigmentation cline.

462 Testing for associations between pigmentation and variation in ebony and tan

464 Although we found few alleles with sequence and/or function equivalent to $D$.

465 novamexicana segregating within $D$. americana, other alleles of tan and/or ebony 466 might still contribute to pigmentation diversity within $D$. americana. To explore this 467 possibility, we tested whether any of the segregating sites sampled in tan

468 (Supplementary Table 6) or ebony (Supplementary Table 7) for our phylogenetic 469 analysis showed a significant association with estimates of pigmentation for each

470 strain (Supplementary Table 8). Specifically, we used a general linear model to test

471 each variable site with a minor allele present in at least five strains (excluding sites

472 with indels) for a statistically significant association with pigmentation. For ebony, the

473 region sampled started in exon 5 and extended into exon 8, with no statistically

474 significant associations observed (Figure 3A). Because prior work suggests that the

475 functional difference between $D$. americana and $D$. novamexicana ebony alleles

476 affects cis-regulation (Cooley et al. 2012), it is perhaps not surprising that this region, 
477 consisting mainly of coding sequences, does not harbor associated variants. We

478 thought it possible, however, that we might have seen an association with these sites

479 due to linkage disequilibrium with a variant outside this region because ebony is

480 located in a region of the genome inverted between $D$. novamexicana and most strains

481 of D. americana (Wittkopp:2003bn; Wittkopp et al. 2009). For tan, prior work has

482 mapped functionally divergent sites to intron 1 (Wittkopp et al. 2009), suggesting that

483 the region sampled is much more likely to harbor variants that might correlate with

484 pigmentation. Nonetheless, we also observed no statistically significant associations

485 between body color and variants in this region segregating within $D$. americana (Figure 486 3B).

Genetic heterogeneity underlying body color variation in D. americana

490 With none of our analyses linking variation in ebony and/or tan to clinal variation in $D$.

491 americana body color, we sought to further investigate its genetic architecture by

492 examining the phenotypic distributions of males in the 51 backcross populations.

493 Because all 51 strains were crossed and then backcrossed to the same strain of $D$.

494 novamexicana, differences in the distribution of pigmentation phenotypes observed

495 among these $\mathrm{BC}_{1}$ populations must be due to genetic differences among the strains of

496 D. americana. For example, differences in the number of phenotypic classes observed

497 among the $\mathrm{BC}_{1}$ populations indicate that different strains of $D$. americana harbor

498 different numbers of loci with effects on pigmentation distinct from the $D$.

499 novamexicana alleles. Assuming basic Mendelian segregation, one locus with a 500 divergent allele affecting pigmentation is expected to cause two distinct pigmentation 
501 phenotypes in the backcross population, whereas two loci with divergent alleles are

502 expected to cause up to four distinct pigmentation phenotypes, and three loci with

503 divergent alleles could cause up to eight distinct phenotypes. Differences in the $\mathrm{BC}_{1}$

504 pigmentation phenotypes and/or number of pigmentation categories are also expected

505 to result from variation among the $D$. americana strains in the identity of loci and/or

506 allelic variation at loci.

507

508 To compare the distributions of $\mathrm{BC}_{1}$ phenotypes among strains, we first converted the

509 strain-specific pigmentation categories to a standardized set of pigmentation

510 categories. We did this by comparing representative images of flies from each strain-

511 specific category to each other and sorting the images with the most similar

512 pigmentation into the same category. This process resulted in 8 categories. After

513 translating the numbers of flies from the strain-specific categories to the standardized

514 categories (Supplementary Table 3), we examined the distribution of flies among

515 pigmentation classes for all of the strains. We found that the number of pigmentation

516 categories in the $\mathrm{BC}_{1}$ population ranged from 4 (e.g., BU0624) to 8 (WS0712) among

517 the strains (Supplementary Table 3; Figure 4A), indicating that the number of loci

518 harboring variation affecting pigmentation is variable within $D$. americana. In addition,

519 even for strains that produced the same number of phenotypic classes in the

520 backcross population, differences were observed in the specific pigmentation

521 phenotypes of each class, indicating that there are also differences in the specific loci

522 or alleles affecting pigmentation between strains. An example of this can be seen by

523 comparing strains BU0624 and PM9936: both strains produced backcross populations 
524 with 4 pigmentation classes, but flies with light pigmentation were common in the

525 BU0624 backcross and nonexistent in the PM9936 backcross (Figure 4A).

527 Finally, we asked whether loci affecting pigmentation were more likely to be more 528 similar for strains isolated from the same population than from different populations.

529 Despite evidence of extensive gene flow within D. americana (Schäfer et al. 2006;

530 Morales-Hojas et al. 2008; Fonseca et al. 2013), we expected this might be true for loci

531 affecting pigmentation because of the longitudinal cline previously observed for body

532 color (Wittkopp et al. 2011). That is, if natural selection is favoring different

533 pigmentation phenotypes in different populations, we might expect to see more genetic

534 similarity for loci affecting pigmentation within than between populations. Inspecting the

535 number of backcross pigmentation categories for strains derived from the same

536 collection site, however, already suggests this might not be so: the three strains

537 isolated from the MK population produced backcross progeny with 4, 6, and 7 distinct 538 pigmentation phenotypes.

540 To further compare the backcross phenotypes, we used correspondence analysis (CA)

541 to reduce the dimensionality of the $\mathrm{BC}_{1}$ phenotypic distributions. This method is similar

542 to principal components analysis (PCA), but for categorical data. The first two

543 dimensions of the correspondence analysis (comparable to the first two principle

544 components in a PCA) captured $55.1 \%$ of the variation among strains. As seen by the

545 overlaid pigmentation categories in Figure 4B, dimension 1 discriminates most strongly

546 between strains that do and do not produce many backcross progeny with the darkest

547 pigmentation (categories 7 and 8). Dimension 2, by contrast, discriminates most 
strongly between strains that do and do not produce many backcross progeny with the

549 lightest pigmentation (categories 1 and 2) (Figure 4B). The lack of visible clustering for

550 strains isolated from the same collection site again suggests that flies in the same

551 population might not be more likely to have similar loci affecting pigmentation than flies

552 from different populations. Indeed, Euclidean distances in this CA dimension 1 and 2

553 space were similar for the 110 pairs of strains from the same collection site and the

5542440 pairs of strains that were from different collection sites (mean distance for pair

555 from same collection site $=0.68$; mean distance for pairs from different collection sites

$556=0.65 ;$ t-test, $\mathrm{p}$-value $=0.45)$.

\section{Discussion}

560 In this study, we tested the hypothesis that $D$. novamexicana-like alleles of ebony

561 and/or tan are driving the longitudinal pigmentation cline seen in $D$. americana

562 (Wittkopp et al. 2009; 2011; Cooley et al. 2012). We found no support for this

563 hypothesis: $D$. novamexicana-like alleles of these genes segregating in $D$. americana -

564 identified based on either sequence or function - were too rare to account for the cline.

565 Other alleles of tan and/or ebony might contribute to pigmentation variation within $D$.

566 americana, but we found no statistically significant association between body color and

567 any of the variable sites in tan or ebony tested. Rather, genetic analysis indicated that

568 differences in the number of loci and/or allelic effects of loci affecting pigmentation are

569 common both within and among populations, suggesting genetic heterogeneity despite

570 locally adapted pigmentation. Below, we discuss the implications of these findings,

571 focusing on possible sources of pigmentation variation in D. americana, the complexity 
572 of its genetic architecture, and how this pigmentation cline might persist in the face of

573 ongoing gene flow.

574

575 In other Drosophila species, differences in body pigmentation segregating within a species have been shown to be associated with variable sites in pigmentation genes,

577 including ebony (Pool \& Aquadro 2007; Takahashi et al. 2007; Rebeiz et al. 2009;

578 Telonis-Scott et al. 2011; Takahashi \& Takano-Shimizu 2011; Bastide et al. 2013;

579 Johnson et al. 2015; Miyagi et al. 2015; Telonis-Scott \& Hoffmann 2018) and tan

580 (Bastide et al. 2013; Yassin et al. 2016; Endler et al. 2016). Despite the lack of

581 associations observed in the current study, we still think it likely that variation in ebony,

582 tan, and/or other pigmentation genes also contribute to pigmentation variation within $D$.

583 americana. We tested for associations between pigmentation and variable sites in

584 ebony and tan using 100 strains each, but larger sample sizes would provide greater

585 power to detect variants with small effects. In addition, we only tested segregating sites

586 in the first intron of tan and in a region starting in exon 5 and ending in exon 8 for ebony.

587 Because linkage disequilibrium in D. americana decays quickly within these genes

588 (often disappearing within 50 bp) (Wittkopp et al. 2009; 2011), it is unlikely that the

589 sites tested would detect functional variants outside of these regions; variable sites in

590 other regions of tan and/or ebony might be found to be associated with $D$. americana

591 body color in future studies.

593 Association studies can also fail to identify genes contributing to trait variation when

594 there is genetic heterogeneity (i.e., multiple genotypes giving rise to the same

595 phenotype) (Korte \& Farlow 2013; Manchia et al. 2013). Genetic heterogeneity is 
expected to be more common for polygenic than single-gene traits, but even when there

597 is only one gene controlling a trait, allelic heterogeneity (multiple alleles with the same

598 phenotypic effects) can still obscure associations with the gene (Savolainen et al. 2013).

599 Our genetic analysis provides two lines of evidence for such heterogeneity underlying

600 pigmentation variation in $D$. americana. First, for tan, we identified six $D$. americana

601 alleles showing at least marginal evidence of similarity between $D$. americana and $D$.

602 novamexicana, indicating that they lighten pigmentation more than other D. americana

603 tan alleles, but these alleles were derived from five different collection sites in four

604 different states (Alabama, Arkansas, Indiana, and Missouri) and in only one case were

605 two of these alleles sampled from the same collection site. This finding suggests that

606 the similar pigmentation of strains collected from these sites exists despite differences

607 in the pigmentation alleles they carry. A similar pattern was reported previously for $D$.

608 americana when a D. novamexicana-like ebony allele causing lighter pigmentation was

609 found to be present in one of three strains with similar pigmentation derived from

610 Duncan, Nebraska (Wittkopp et al. 2009). Indeed, these D. novamexicana-like tan and

611 ebony alleles found segregating in $D$. americana provide an excellent example of how

612 genetic heterogeneity can work: because ebony and tan encode enzymes catalyzing

613 opposite directions of a reversible biochemical reaction (True et al. 2005), alleles

614 increasing activity of ebony and decreasing activity of tan (or vice versa) can have

615 equivalent effects on pigmentation (Figure 1B, (Wittkopp et al. 2009).

617 Our phenotypic analysis of backcross populations from 51 strains of $D$. americana from

61820 collection sites provides the second line of evidence for genetic heterogeneity

619 underlying clinally varying pigmentation in D. americana. In the absence of genetic 
620 heterogeneity, two strains derived from the same population with the same phenotype

621 are expected to carry the same pigmentation alleles. If true, crossing and backcrossing

622 these strains of $D$. americana to $D$. novamexicana should produce the same

623 distributions of pigmentation phenotypes. We found, however, that backcross

624 populations often showed differences in the number of distinct pigmentation classes, the

625 body color of each pigmentation class, and/or the relative abundance of flies with

626 different body colors, even when strains were derived from the same collection site.

627 These data are consistent with genetic heterogeneity in which multiple combinations of

628 genes and/or alleles underlie similar pigmentation phenotypes within a population as

629 well as diversity in pigmentation among locations. Similar genetic heterogeneity has

630 previously been described for mate choice in Drosophila pseudoobscura (Barnwell \&

631 Noor 2008), gene expression in yeast (Metzger \& Wittkopp 2019), timing of bud set in

632 Scots pine trees (Kujala et al. 2017), flowering time in maize (Buckler et al. 2009), and

633 human diseases (McClellan \& King 2010). It has also been reported more broadly for

634 convergent phenotypes that evolved in more genetically isolated populations, including

635 adaptation of humans to high-altitudes (Jeong \& Di Rienzo 2014), lighter skin color in

636 East Asian and European peoples (Norton et al. 2007), and adaptation to highlands in

637 maize (Takuno et al. 2015). Nonetheless, we think that the extent of genetic

638 heterogeneity underlying variation in quantitative traits is generally underestimated -

639 especially within a population or among populations connected by extensive gene flow -

640 because of the reliance on association mapping for finding loci responsible for trait

641 variation and the rarity of studies using biparental quantitative trait locus (QTL) mapping

642 to analyze multiple genotypes from the same population with similar phenotypes. 
644 How might this genetic complexity be maintained despite selection favoring a particular

645 phenotype at a particular location? The extensive gene flow seen throughout $D$.

646 americana (Schäfer et al. 2006; Morales-Hojas et al. 2008; Wittkopp et al. 2011;

647 Fonseca et al. 2013) is likely part of the answer. This gene flow moves alleles among 648 populations, making it difficult for a population to fix the most adaptive allele for each

649 local environment (Savolainen et al. 2013). But there must also be sufficient genetic

650 variation affecting pigmentation maintained in the species for this gene flow to cause

651 genetic heterogeneity (Pritchard et al. 2010; Savolainen et al. 2013). D. americana

652 harbors high levels of genetic variation generally (Fonseca et al. 2013), and selection

653 for different pigmentation phenotypes in different locations should maintain diverse

654 pigmentation alleles at the species level (Savolainen et al. 2013; Lee et al. 2016; Troth

655 et al. 2018). The structure of the biochemical pathway controlling production of

656 alternative pigments from a single, branched biochemical pathway (Massey \& Wittkopp

657 2016) might also contribute to standing genetic variation because it allows changes in

658 the activity of multiple genes to have similar effects on pigmentation (Wittkopp et al.

659 2009). Ultimately, however, selection acting on this standing genetic variation must be

660 favoring different pigmentation phenotypes in different locations to maintain the cline

661 (Kawecki \& Ebert 2004; Savolainen et al. 2013). Assortative mating, in which individuals

662 with similar body color are more likely to mate with each other than individuals with

663 different body color, could also contribute to the D. americana pigmentation cline.

664 Although evidence of assortative mating for body color is rare in Drosophila species, it

665 has been observed in an Indian population of $D$. melanogaster, with darker individuals

666 more likely to mate with each other in cold, dry weather and lighter individuals more

667 likely to mate with each other when it is hot or humid (Dev et al. 2013). Disentangling 
the relative contributions of these different evolutionary and molecular processes to the

669 formation and maintenance of the $D$. americana body color cline will require much more

670 extensive, interdisciplinary studies.

Acknowledgements

675 We thank Bryant McAllister for sharing strains of $D$. americana that his lab established

676 from field collections, the Drosophila Species Stock Center for maintaining and

677 supplying strains of D. novamexicana and D. americana, and members of the Wittkopp

678 lab (especially Henry Ertl, Mark Hill, Petra Vande Zande, Abigail Lamb and Molly Hirst)

679 for helpful discussions and feed-back on this work. Funding for this project was provided

680 by the National Institutes of Health (F32GM087928 to AC; R35GM118073 and

681 R01GM08973 to PJW) and the National Science Foundation (DEB-0640485 to PJW).

\section{References}

684

685
Adrion JR, Hahn MW, Cooper BS (2015) Revisiting classic clines in Drosophila melanogaster in the age of genomics. Trends in Genetics, 31, 434-444.

Ahmadian A, Lundeberg J, Nyrén P, Uhlén M, Ronaghi M (2000) Analysis of the p53 tumor suppressor gene by pyrosequencing. BioTechniques, 28, 140-147.

Ahmed-Braimah YH, McAllister BF (2012) Rapid Evolution of Assortative Fertilization between Recently Allopatric Species of Drosophila. International Journal of Evolutionary Biology, 2012, 285468-9.

Bandelt HJ, Forster P, Röhl A (1999) Median-joining networks for inferring intraspecific phylogenies. Molecular Biology and Evolution, 16, 37-48.

Barnwell CV, Noor MAF (2008) Failure to Replicate Two Mate Preference QTLs across Multiple Strains of Drosophila pseudoobscura. The Journal of Heredity, 99, 653656.

Bastide H, Betancourt A, Nolte $V$ et al. (2013) A genome-wide, fine-scale map of natural pigmentation variation in Drosophila melanogaster. PLoS Genetics, 9, e1003534. 
699

700

701

702

703

704

705

706

707

708

709

710

711

712

713

714

715

716

717

718

719

720

721

722

723

724

725

726

727

728

729

730

731

732

733

734

735

736

737

738

739

740

741

742

743
Bastide H, Yassin A, Johanning EJ, Pool JE (2014) Pigmentation in Drosophila melanogaster reaches its maximum in Ethiopia and correlates most strongly with ultra-violet radiation in sub-Saharan Africa. BMC Evolutionary Biology, 14, 179.

Benjamini, Y., \& Hochberg, Y. (1995). Controlling the false discovery rate: a practical and powerful approach to multiple testing. Journal of the Royal Statistical Society Series $B, 57(1), 289-300$.

Brisson JA, De Toni DC, Duncan I, Templeton AR (2005) Abdominal pigmentation variation in Drosophila polymorpha: geographic variation in the trait, and underlying phylogeography. Evolution, 59, 1046-1059.

Buckler ES, Holland JB, Bradbury PJ et al. (2009) The Genetic Architecture of Maize Flowering Time. Science, 325, 714-718.

Caletka BC, McAllister BF (2004) A genealogical view of chromosomal evolution and species delimitation in the Drosophila virilis species subgroup. Molecular Phylogenetics and Evolution, 33, 664-670.

Clusella-Trullas S, Terblanche JS (2011) Local adaptation for body color in Drosophila americana: commentary on Wittkopp et al. Heredity, 106, 904-905.

Cooley AM, Shefner L, McLaughlin WN, Stewart EE, Wittkopp PJ (2012) The ontogeny of color: developmental origins of divergent pigmentation in Drosophila americana and D. novamexicana. Evolution \& Development, 14, 317-325.

David JR, Capy P (1988) Genetic variation of Drosophila melanogaster natural populations. Trends in Genetics, 4, 106-111.

David, J. R., Capy, P., Payant, V., \& Tsakas, S. (1985). Thoracic trident pigmentation in Drosophila melanogaster: differentiation of geographical populations. Genet Sel Evol, 17, 211-224.

Davis JS, Moyle LC (2019) Desiccation resistance and pigmentation variation reflects bioclimatic differences in the Drosophila americana species complex. BMC Evolutionary Biology, 19, 204-14.

Dev K, Chahal J, Parkash R (2013) Seasonal variations in the mating-related traits of Drosophila melanogaster. Journal of Ethology, 31, 165-174.

Endler JA (1977) Geographic Variation, Speciation and Clines. Princeton University Press, Princeton, NJ.

Endler L, Betancourt AJ, Nolte V, Schlötterer C (2016) Reconciling Differences in PoolGWAS Between Populations: A Case Study of Female Abdominal Pigmentation in Drosophila melanogaster. Genetics, 202, 843-855.

Felsenstein J (1985) Confidence Limits on Phylogenies: an Approach Using the Bootstrap. Evolution, 39, 783-791.

Fonseca NA, Morales-Hojas R, Reis M et al. (2013) Drosophila americana as a model species for comparative studies on the molecular basis of phenotypic variation. Genome Biology and Evolution, 5, 661-679.

Fournier-Level A, Korte A, Cooper MD et al. (2011) A Map of Local Adaptation in Arabidopsis thaliana. Science, 334, 86-89.

François O, Martins H, Caye K, Schoville SD (2016) Controlling false discoveries in genome scans for selection. Molecular Ecology, 25, 454-469.

Gloor GB, Preston CR, Johnson-Schlitz DM et al. (1993) Type I repressors of P element mobility. Genetics, 135, 81-95. 
744

753

754

755

756

757

758

759

760

761

762

763

764

765

766

767

768

769

770

771

772

773

774

775

776

777

778

779

780

781

782

783

784

785

786

787

788

Haasl RJ, Payseur BA (2016) Fifteen years of genomewide scans for selection: trends, lessons and unaddressed genetic sources of complication. Molecular Ecology, 25, 5-23.

Hancock AM, Brachi B, Faure N et al. (2011) Adaptation to Climate Across the Arabidopsis thaliana Genome. Science, 334, 83-86.

Hollocher H, Hatcher JL, Dyreson EG (2000) Evolution of abdominal pigmentation differences across species in the Drosophila dunni subgroup. Evolution, 54, 20462056.

Huxley J (1938) Clines: an auxiliary taxonomic principle. Nature, 142, 219-220.

Jeong C, Di Rienzo A (2014) Adaptations to local environments in modern human populations. Current Opinion in Genetics \& Development, 29, 1-8.

Johnson WC, Ordway AJ, Watada M et al. (2015) Genetic Changes to a Transcriptional Silencer Element Confers Phenotypic Diversity within and between Drosophila Species. PLoS Genetics, 11, e1005279.

Kawecki TJ, Ebert D (2004) Conceptual issues in local adaptation. Ecology Letters, 7, 1225-1241.

Korte A, Farlow A (2013) The advantages and limitations of trait analysis with GWAS: a review. Plant Methods, 9, 29.

Kujala ST, Knürr T, Kärkkäinen K et al. (2017) Genetic heterogeneity underlying variation in a locally adaptive clinal trait in Pinus sylvestris revealed by a Bayesian multipopulation analysis. Heredity, 118, 413-423.

Kumar S, Stecher G, Tamura K (2016) MEGA7: Molecular Evolutionary Genetics Analysis Version 7.0 for Bigger Datasets. Molecular Biology and Evolution, 33, 1870-1874.

Lamb AM, Wang Z, Simmer P, Chung H, Wittkopp PJ (2020) ebony affects pigmentation divergence and cuticular hydrocarbons in Drosophila americana and D. novamexicana. bioRxiv, 57, 2020.03.05.977009.

Lê, S., Josse, J., \& Husson, F. (2008). FactoMineR: An RPackage for Multivariate Analysis. Journal of Statistical Software, 25(1), 1-18.

Lee YW, Fishman L, Kelly JK, Willis JH (2016) A Segregating Inversion Generates Fitness Variation in Yellow Monkeyflower (Mimulus guttatus). Genetics, 202, 14731484.

Lotterhos KE, Whitlock MC (2015) The relative power of genome scans to detect local adaptation depends on sampling design and statistical method. Molecular Ecology, 24, 1031-1046.

Manchia M, Cullis J, Turecki G et al. (2013) The Impact of Phenotypic and Genetic Heterogeneity on Results of Genome Wide Association Studies of Complex Diseases. PloS ONE, 8, e76295.

Marjoram P, Zubair A, Nuzhdin SV (2013) Post-GWAS: where next? More samples, more SNPs or more biology? Heredity, 112, 79-88.

Massey JH, Wittkopp PJ (2016) The Genetic Basis of Pigmentation Differences Within and Between Drosophila Species. Current Topics in Developmental Biology, 119, 27-61.

Matute DR, Harris A (2013) The influence of abdominal pigmentation on desiccation and ultraviolet resistance in two species of Drosophila. Evolution, 67, 2451-2460. 
McAllister, B. F., Sheeley, S. L., Mena, P. A., Evans, A. L., \& Schlötterer, C. (2008). Clinal distribution of a chromosomal rearrangement: a precursor to chromosomal speciation? Evolution, 62(8), 1852-1865.

McClellan J, King M-C (2010) Genetic heterogeneity in human disease. Cell, 141, 210217.

Metzger BPH, Wittkopp PJ (2019) Compensatory trans-regulatory alleles minimizing variation in TDH3 expression are common within Saccharomyces cerevisiae. Evolution letters, 3, 448-461.

Miyagi R, Akiyama N, Osada N, Takahashi A (2015) Complex patterns of cis-regulatory polymorphisms in ebony underlie standing pigmentation variation in Drosophila melanogaster. Molecular Ecology, 24, 5829-5841.

Morales-Hojas R, Vieira CP, Vieira J (2008) Inferring the evolutionary history of Drosophila americana and Drosophila novamexicana using a multilocus approach and the influence of chromosomal rearrangements in single gene analyses. Molecular Ecology, 17, 2910-2926.

Norton HL, Kittles RA, Parra E et al. (2007) Genetic evidence for the convergent evolution of light skin in Europeans and East Asians. Molecular Biology and Evolution, 24, 710-722.

Orsini L, Huttunen S, Schlötterer C (2004) A multilocus microsatellite phylogeny of the Drosophila virilis group. Heredity, 93, 161-165.

Parkash R, Aggarwal DD, Ranga P, Singh D (2012) Divergent strategies for adaptation to desiccation stress in two Drosophila species of immigrans group. Journal of comparative physiology. B, Biochemical, systemic, and environmental physiology, 182, 751-769.

Pool JE, Aquadro CF (2007) The genetic basis of adaptive pigmentation variation in Drosophila melanogaster. Molecular Ecology, 16, 2844-2851.

Pritchard JK, Di Rienzo A (2010) Adaptation - not by sweeps alone. Nature Reviews Genetics, 11, 665-667.

Pritchard JK, Pickrell JK, Coop G (2010) The Genetics of Human Adaptation: Hard Sweeps, Soft Sweeps, and Polygenic Adaptation. Current Biology, 20, R208-R215.

Rajpurohit S, Schmidt PS (2019) Latitudinal Pigmentation Variation Contradicts Ultraviolet Radiation Exposure: A Case Study in Tropical Indian Drosophila melanogaster. Frontiers in physiology, 10, 84.

Rajpurohit S, Parkash R, Ramniwas S (2008) Body melanization and its adaptive role in thermoregulation and tolerance against desiccating conditions in drosophilids. Entomological Research, 38, 49-60.

Rebeiz M, Pool JE, Kassner VA, Aquadro CF, Carroll SB (2009) Stepwise modification of a modular enhancer underlies adaptation in a Drosophila population. Science, 326, 1663-1667.

Savolainen O, Lascoux M, Merilä J (2013) Ecological genomics of local adaptation. Nature Reviews Genetics, 14, 807-820.

Schäfer MA, Orsini L, McAllister BF, Schlötterer C (2006) Patterns of microsatellite variation through a transition zone of a chromosomal cline in Drosophila americana. Heredity, 97, 291-295.

Sheeley SL, McAllister BF (2008) Patterns of natural selection at the Alcohol dehydrogenase gene of Drosophila americana. Fly, 2, 243-246. 
835

836

837

838

839

840

841

842

843

844

845

846

Sillero N, Reis M, Vieira CP, Vieira J, Morales-Hojas R (2014) Niche evolution and thermal adaptation in the temperate species Drosophila americana. Journal of Evolutionary Biology, 27, 1549-1561.

Stinchcombe JR, Hoekstra HE (2007) Combining population genomics and quantitative genetics: finding the genes underlying ecologically important traits. Heredity, 100, 158-170.

Takahashi A, Takano-Shimizu T (2011) Divergent enhancer haplotype of ebony on inversion $\ln (3 R)$ Payne associated with pigmentation variation in a tropical population of Drosophila melanogaster. Molecular Ecology, 20, 4277-4287.

Takahashi A, Takahashi K, Ueda R, Takano-Shimizu T (2007) Natural variation of ebony gene controlling thoracic pigmentation in Drosophila melanogaster. Genetics, 177, 1233-1237.

Takuno S, Ralph P, Swarts K et al. (2015) Independent Molecular Basis of Convergent Highland Adaptation in Maize. Genetics, 200, 1297-1312.

Tamura K, Nei M (1993) Estimation of the number of nucleotide substitutions in the control region of mitochondrial DNA in humans and chimpanzees. Molecular Biology and Evolution, 10, 512-526.

Telonis-Scott M, Hoffmann AA (2018) Enhancing Ebony? Common Associations With a cis-Regulatory Haplotype for Drosophila melanogaster Thoracic Pigmentation in a Japanese Population and Australian Populations. Frontiers in physiology, 9, 822.

Telonis-Scott M, Hoffmann AA, Sgro CM (2011) The molecular genetics of clinal variation: a case study of ebony and thoracic trident pigmentation in Drosophila melanogaster from eastern Australia. Molecular Ecology, 20, 2100-2110.

Thompson JD, Higgins DG, Gibson TJ (1994) CLUSTAL W: improving the sensitivity of progressive multiple sequence alignment through sequence weighting, positionspecific gap penalties and weight matrix choice. Nucleic Acids Research, 22, 46734680.

Troth A, Puzey JR, Kim RS, Willis JH, Kelly JK (2018) Selective trade-offs maintain alleles underpinning complex trait variation in plants. Science, 361, 475-478.

True JR (2003) Insect melanism: the molecules matter. Trends in Ecology \& Evolution, 18, 640-647.

True JR, Yeh S-D, Hovemann BT et al. (2005) Drosophila tan encodes a novel hydrolase required in pigmentation and vision. PLoS Genetics, 1, e63.

Wittkopp PJ, Beldade P (2009) Development and evolution of insect pigmentation: genetic mechanisms and the potential consequences of pleiotropy. Seminars in Cell \& Developmental Biology, 20, 65-71.

Wittkopp PJ, Haerum BK, Clark AG (2004) Evolutionary changes in cis and trans gene regulation. Nature, 430, 85-88.

Wittkopp PJ, Haerum BK, Clark AG (2008) Regulatory changes underlying expression differences within and between Drosophila species. Nature Genetics, 40, 346-350.

Wittkopp PJ, Smith-Winberry G, Arnold LL et al. (2011) Local adaptation for body color in Drosophila americana. Heredity, 106, 592-602.

Wittkopp PJ, Stewart EE, Arnold LL et al. (2009) Intraspecific polymorphism to interspecific divergence: genetics of pigmentation in Drosophila. Science, 326, 540544. 
880 Wittkopp PJ, Williams BL, Selegue JE, Carroll SB (2003) Drosophila pigmentation evolution: divergent genotypes underlying convergent phenotypes. Proc. Natl. Acad. Sci. U.S.A., 100, 1808-1813.

Yassin A, Bastide $\mathrm{H}$, Chung $\mathrm{H}$ et al. (2016) Ancient balancing selection at tan underlies female colour dimorphism in Drosophila erecta. Nature communications, 7, 10400.

\section{Data Accessibility}

887 Sequences described in Supplementary Files 1 and 2 are also available in NCBI

888 PopSet with GenBank accession numbers: MT350927 - MT351036 for ebony and

MT350824 - MT350926 for tan. All other data and code are included in the manuscript

890 as supplementary tables and files.

\section{Author Contributions}

893 PJW and LLS designed the research. LLS and WNM quantified pigmentation and

894 performed the genetic analysis. AMC, DCY, AJ, and PJW collected and analyzed

895 sequence data. PJW performed the statistical analysis and constructed figures, with

896 assistance from LLS and WNM. PJW wrote the paper, with input from LLS, WNM, and

897 AJ and final editing by all authors. 

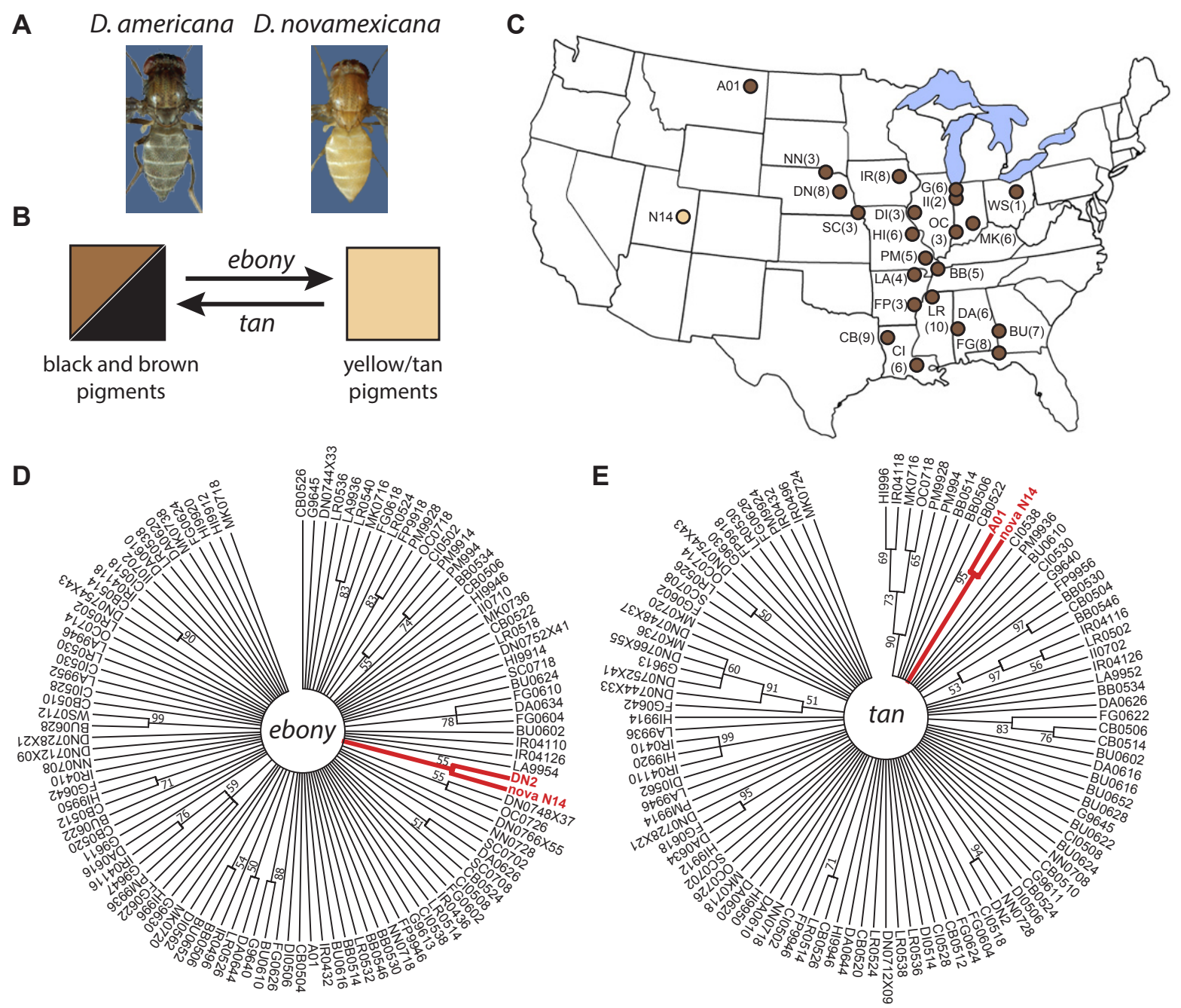

Figure 1. D. americana alleles of ebony and tan closely related to the $D$. novamexicana allele are rare within $D$. americana. (A) $D$. americana (left) has a much darker body color than $D$. novamexicana (right). (B) The tan and ebony genes encode enzymes that catalyze a reversible biochemical reaction required for the production of dark (black and brown) melanins and light (yellow/tan) sclerotins, respectively. (C) Collection sites for progenitors of $D$. americana (brown) and $D$. novamexicana (yellow) strains used in this work are shown. Numbers in parentheses indicate the number of independently isolated strains examined from that site. Only a single strain from the Drosophila Species Stock Center was examined for A01 and N14. For more information about these strains, see Supplementary Table 1. (D, E) The circular phylogenetic trees shown for ebony $(\mathrm{D})$ and $\tan (\mathrm{E})$ were produced using a Maximum Likelihood method implemented in MEGA7, as described in Methods. Branches shown were supported by $50 \%$ or more of bootstrap replicate trees. The ebony tree is based on 579 aligned sites from 110 alleles, and the tan tree is based on 1328 aligned sites from 103 alleles. Branches shown in red highlight the $D$.

novamexicana allele ("nova N14") and the allele from D. americana (DN2 for ebony, A01 for tan) previously shown to share similarity in both sequence and function with the $D$. novamexicana allele (Wittkopp et al. 2009). 
A

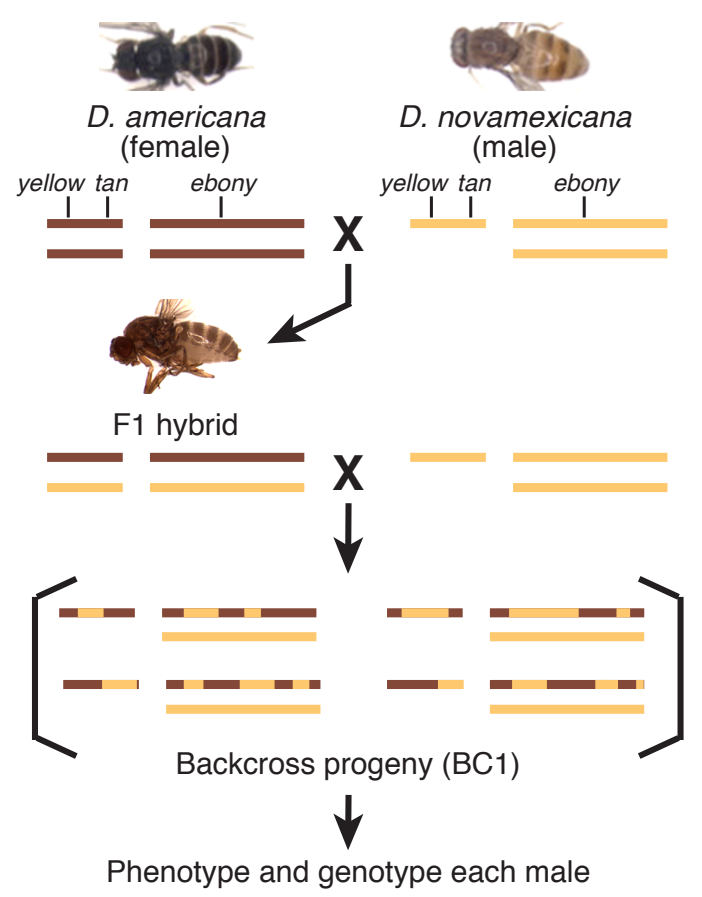

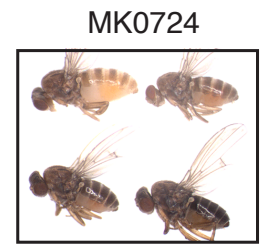

IR04110

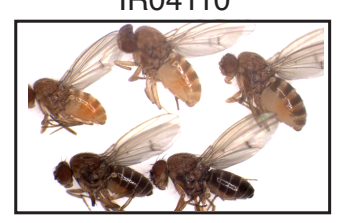

C

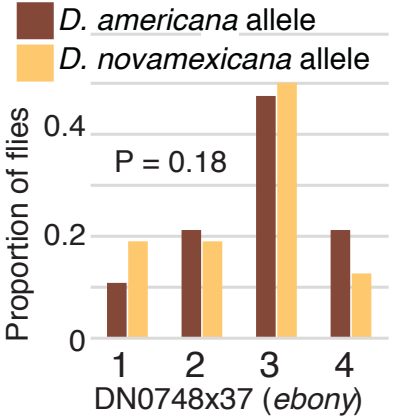

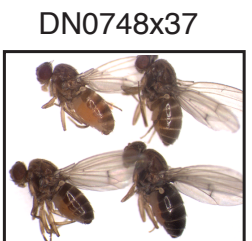

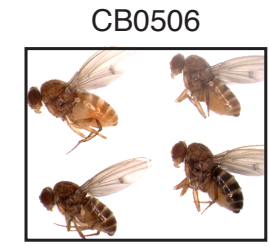

II0702
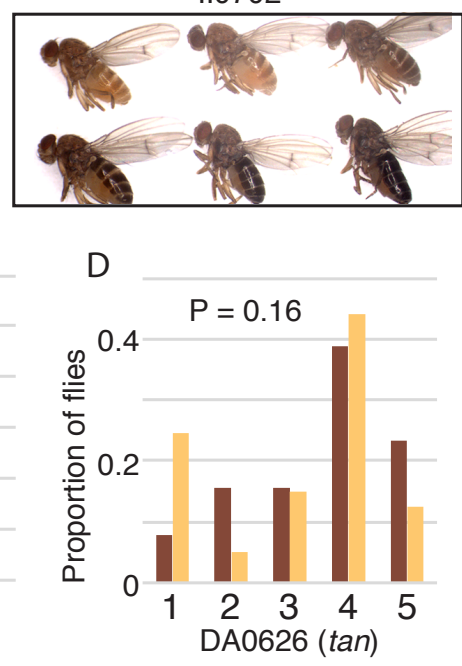

Figure 2. Genetic analysis of pigmentation differences between $D$. novamexicana and strains of $D$. americana. (A) Schematics show chromosomal content of $D$. americana and $D$. novamexicana parental strains, $F_{1}$ hybrids, and examples of potential backcross progeny produced by crossing an $\mathrm{F}_{1}$ hybrid female back to $D$. novamexicana, with all autosomes represented as a single bar. Approximate locations of the yellow and tan genes on the $\mathrm{X}$ chromosome (Muller Element A) as well as the ebony gene on chromosome 2 (Muller element $\mathrm{E}$ ) are also shown. Dorsal images of $D$. novamexicana (strain $\mathrm{N} 14$ ) and D. americana (strain CB0522) as well as the lateral image of a $F_{1}$ hybrid shown were taken at different times from each other and images shown in panel B. Color adjustments have been made to reproduce relative pigmentation of these three genotypes, but these images should not be quantitatively compared to each other or images in panel B. (B) Representative flies from each of the 4 to 6 pigmentation classes identified for five strains of $D$. americana are shown, arranged from lightest (top left) to darkest (bottom right) in each box. A lateral view is shown for all flies and images within a box were collected under comparable conditions. (C, D) The proportion of male backcross flies in each pigmentation class carrying a $D$. americana (brown) or $D$. novamexicana (yellow) allele of ebony (C) or tan (D) inherited from their $F_{1}$ hybrid mother is shown for backcrosses with two strains of D. americana: DN0748x37 (C) and DA0626 (D). These two examples are the only cases where no statistically significant difference in body color was detected for flies inheriting the $D$. americana or $D$. novamexicana alleles of ebony or tan. Phenotypic distributions are shown for yellow, ebony, and tan genotypes for all strains of $D$. americana in Supplementary Figures 3, 4 and 5, respectively. Note that borderline evidence of functional similarity for tan alleles was also observed between $D$. novamexicana and five other strains of $D$. americana (Supplementary Figure 5). None of the $D$. americana strains showed evidence of functional differences from $D$. 
novamexicana for alleles of the yellow gene (Supplementary Figure 3). Genotyping data for all three genes is provided as Supplementary Table 4, and results of the statistical tests are provided as Supplementary Table 5.

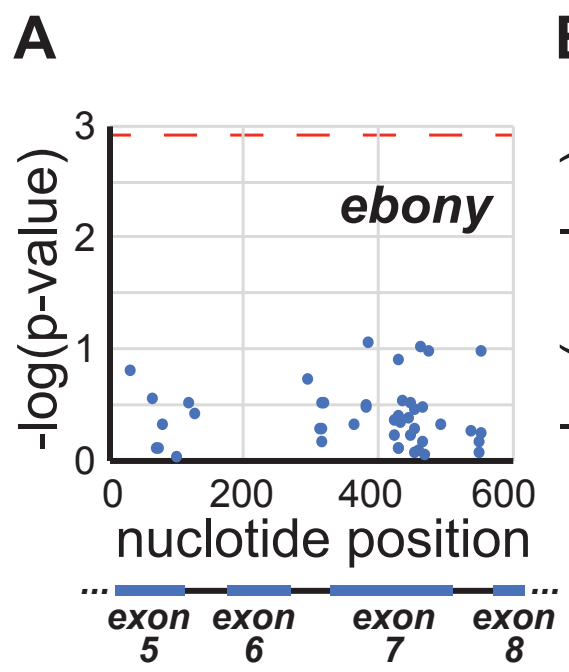

B

Figure 3. Variable sites sampled in tan and ebony are not significantly associated with pigmentation in $\boldsymbol{D}$. americana. Statistical significance of an association between body color and the nucleotide present at variable sites in the $D$. americana ebony $(\mathrm{A})$ and $\tan (\mathrm{B})$ regions sequenced are shown, reported as - $\log (\mathrm{p}$ value) from the general linear model described in Methods. Red dotted lines show threshold used to assess statistical significance. Schematics shown below each plot indicate the location of intronic and exons regions in the ebony (A) and tan (B) sequences analyzed. Body color data used provided as Supplementary Table 2. Genotype data used provided as Supplementary Table 6 for tan and Supplementary Table 7 for ebony. Results of the general linear models are provided as Supplementary Table 8. 


\section{A}

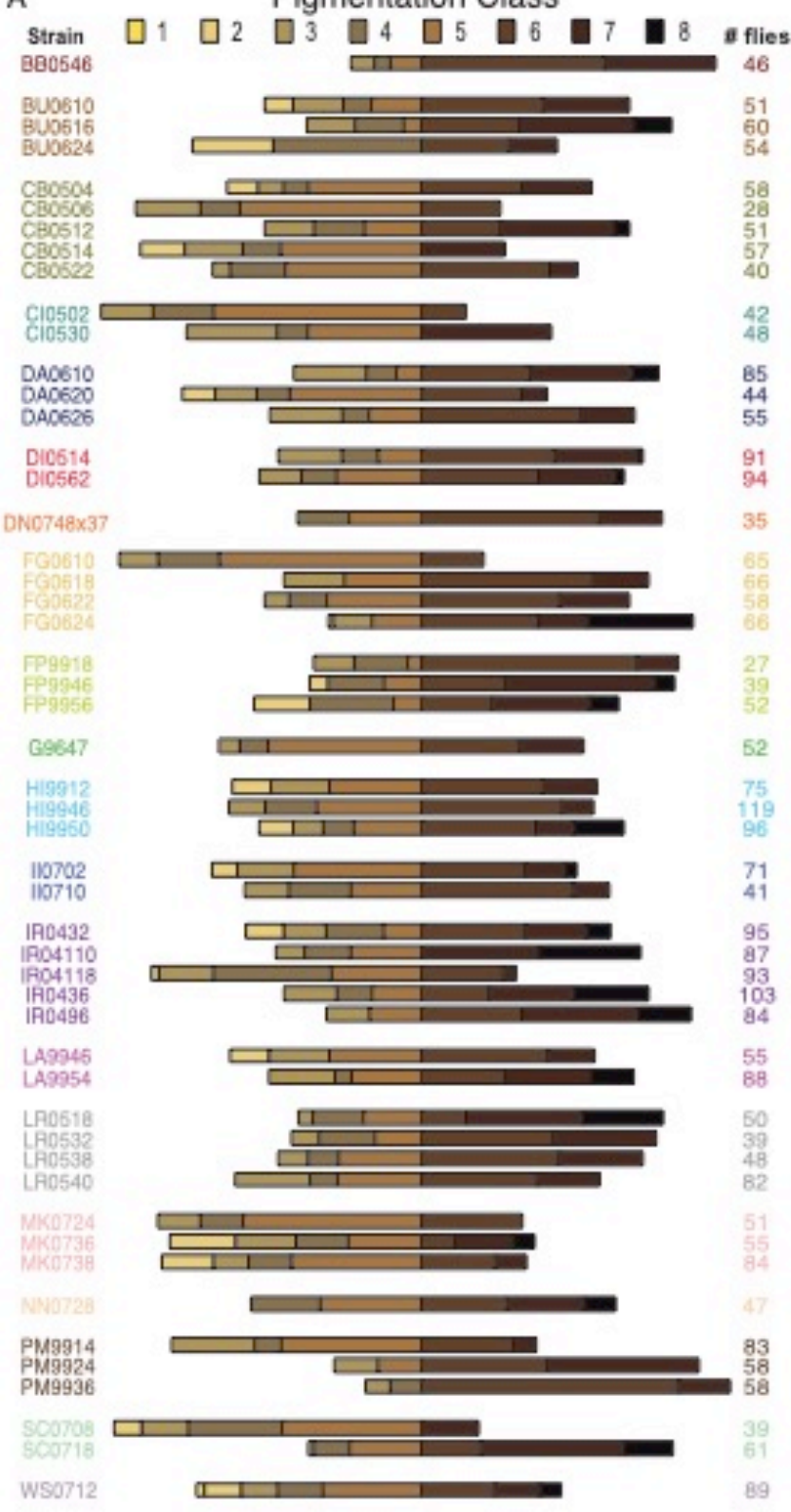

B

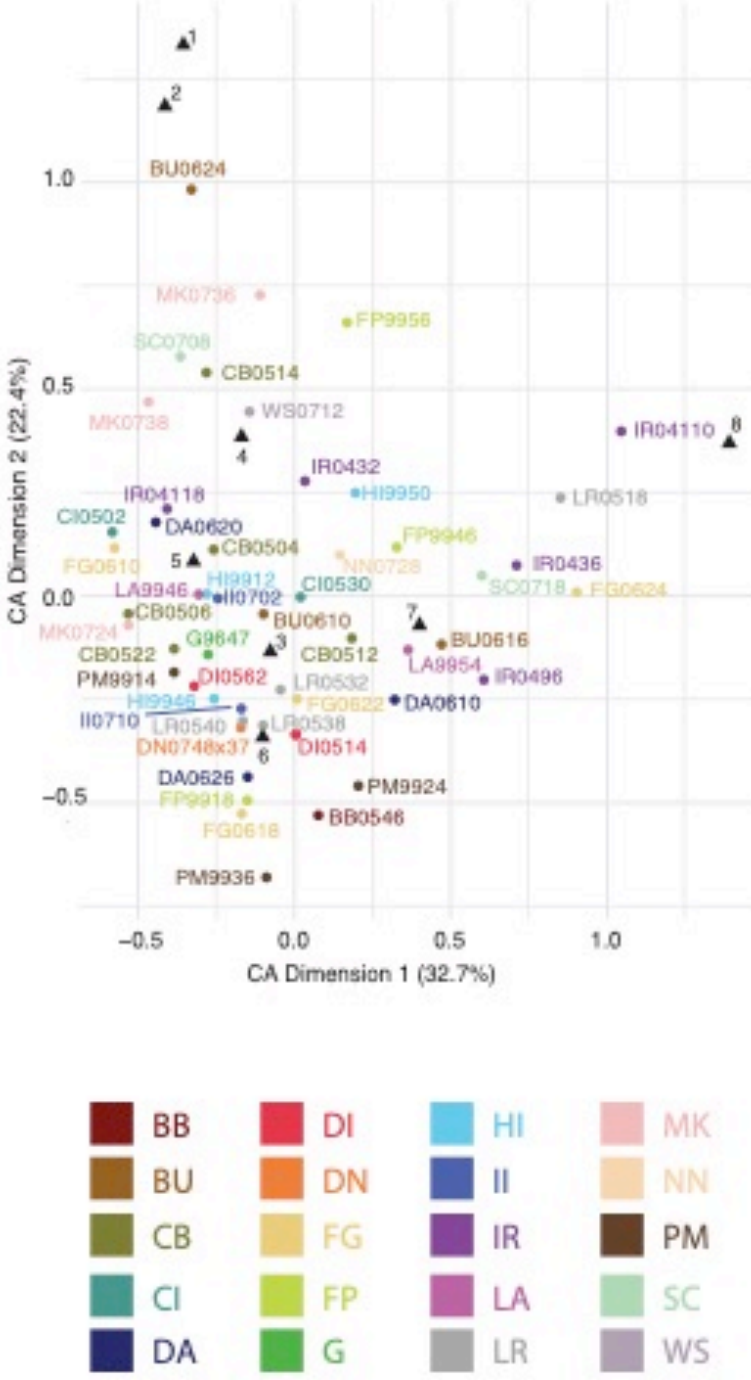

Pigmentation class (1-8)

Figure 4. Distributions of backcross phenotypes indicate diversity in number and effects of loci affecting pigmentation. (A) The relative proportion of male backcross progeny in each of eight standardized pigmentation classes (Supplementary Table 3 ) is shown for each $D$. americana strain. Pigmentation classes are indicated by the color of the bar ranging from the lightest (yellow, class 1 ) to the darkest (black, class 8 ), with a longer bar indicating a greater proportion of the backcross population. Bars are aligned vertically at the transition between pigmentation classes 5 and 6 . Strains are clustered by collection site, with each strain derived from the same collection site shown in the same color. The total number of male backcross progeny scored for each strain is shown to the right of each distribution. Note the differences in distributions not only between, but also within, collection sites. For example, strains producing very different distributions of backcross progeny were isolated from the FG, 
IR, and SC collection sites. (B) Results from a correspondence analysis (CA) used to compare the distribution of backcross pigmentation phenotypes among strains are shown, plotted with colored circles according to their values on the first two axes of variation: CA dimension 1 , which explained $32.7 \%$ of the variation and CA dimension 2 , which explained $22.4 \%$ of the variation. Strains shown with the same color were derived from the same collection site. The relative placement of pigmentation classes 1 - 8 on these two axes is also shown with black triangles for comparison. Note that, for example, strain IR4110, which had most backcross progeny with the darkest body color is located close to the triangle representing the darkest pigmentation class (class 8). Similarly, BU0624, the strain that produced the most lightly pigmented backcross progeny, is located close to the triangles representing the lightest pigmentation classes (class 1 and 2). The lack of visual clustering for strains derived from the same collection site is consistent with our statistical test showing strains from the same collection site were no more likely to be located close to each other in this CA space than strains from different collection sites. 
bioRxiv preprint doi: https://doi.org/10.1101/2020.05.07.074211; this version posted May 9, 2020. The copyright holder for this preprint (which

was not certified by peer review) is the author/funder, who has granted bioRxiv a license to display the preprint in perpetuity. It is made available under aCC-BY 4.0 International license.

\section{Supplemental Information for:}

Genetic architecture of clinal body color variation in Drosophila americana

Lisa L. Sramkoski ${ }^{*}$, Wesley N. McLaughlin*, Arielle M. Cooley, David C. Yuan, Alisha John, and Patricia J. Wittkopp

Table of Contents:

\begin{tabular}{|l|l|}
\hline Supplementary Figure $\mathbf{1}$ & Page $\mathbf{~}$ \\
\hline Supplementary Figure $\mathbf{2}$ & Page 3 \\
\hline Supplementary Figure $\mathbf{3}$ & Page $\mathbf{4}$ \\
\hline Supplementary Figure $\mathbf{4}$ & Page 5 \\
\hline Supplementary Figure $\mathbf{5}$ & Page 6 \\
\hline Summary of Supplementary Tables & Page 7 \\
\hline Summary of Supplementary Files & Page 7 \\
\hline
\end{tabular}




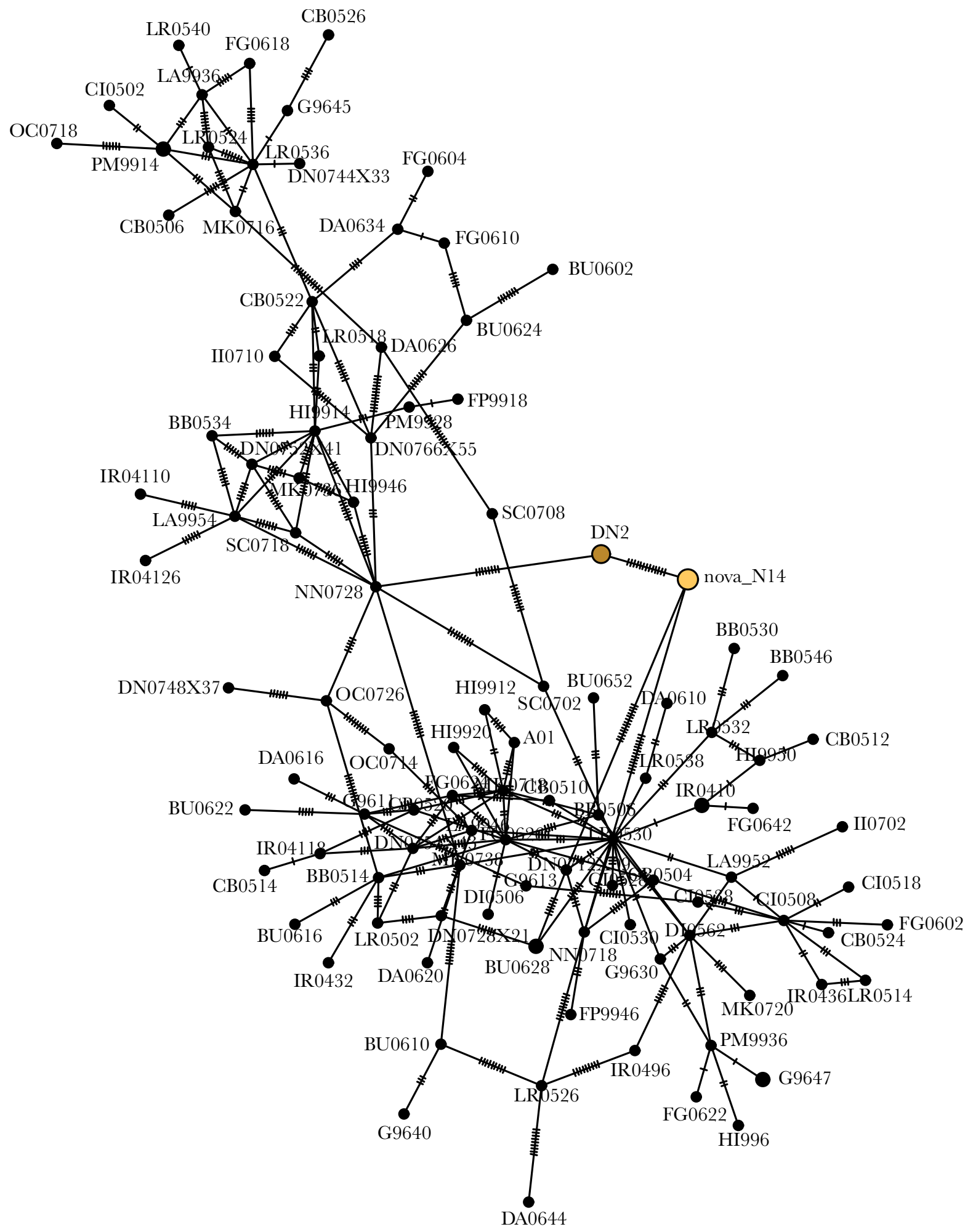

\section{Supplementary Figure 1. Haplotype network for ebony. A Median Spanning Network} built from the same ebony sequences used to construct the phylogenetic tree shown in Figure 1D is shown. Note that the DN2 allele from $D$. americana previously shown to share similarity in sequence and function with $D$. novamexicana (brown) is most similar to the $D$. novamexicana ("nova_N14") allele (yellow). 


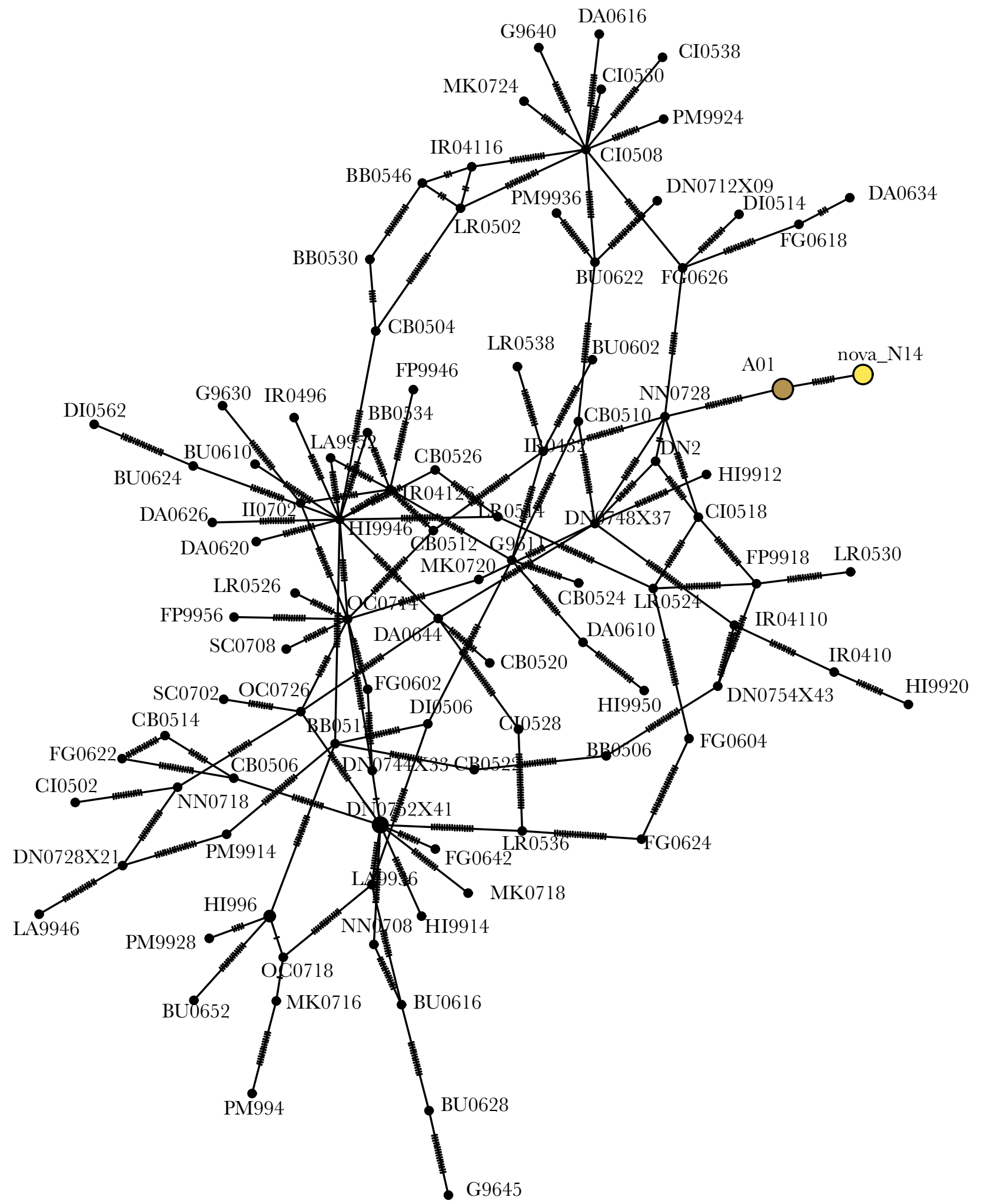

Supplementary Figure 2. Haplotype network for tan. A Median Spanning Network built from the same tan sequences used to construct the phylogenetic tree shown in Figure 1E is shown. Note that the A01 allele from $D$. americana previously shown to share similarity in sequence and function with $D$. novamexicana (brown) is most similar to the $D$. novamexicana ("nova_N14") allele (yellow). 


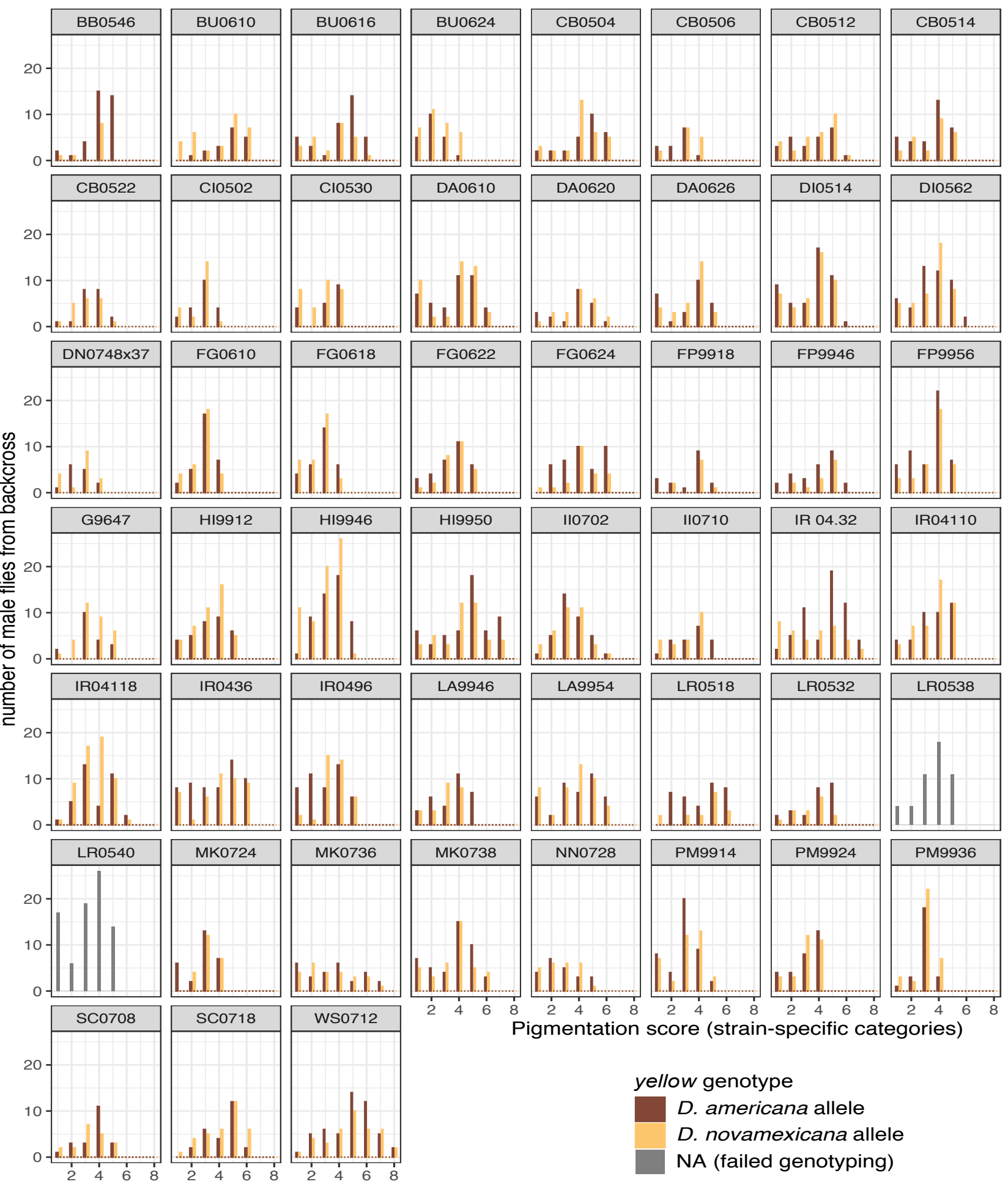

Supplementary Figure 3. Testing for function divergence of yellow. Distributions of pigmentation phenotypes for backcross progeny inheriting the $D$. americana (brown) or $D$. novamexicana allele (yellow) of the yellow gene from their $F_{1}$ hybrid mother are shown for each strain of $D$. americana tested, with the stain name shown at the top of each panel. Numbers of males in each pigmentation class are shown rather than proportions to communicate sample sizes. Grey bars are shown for strains LR0538 and LR0540 because the yellow genotyping assay failed for all flies, presumably because of sequence differences in these yellow alleles. 


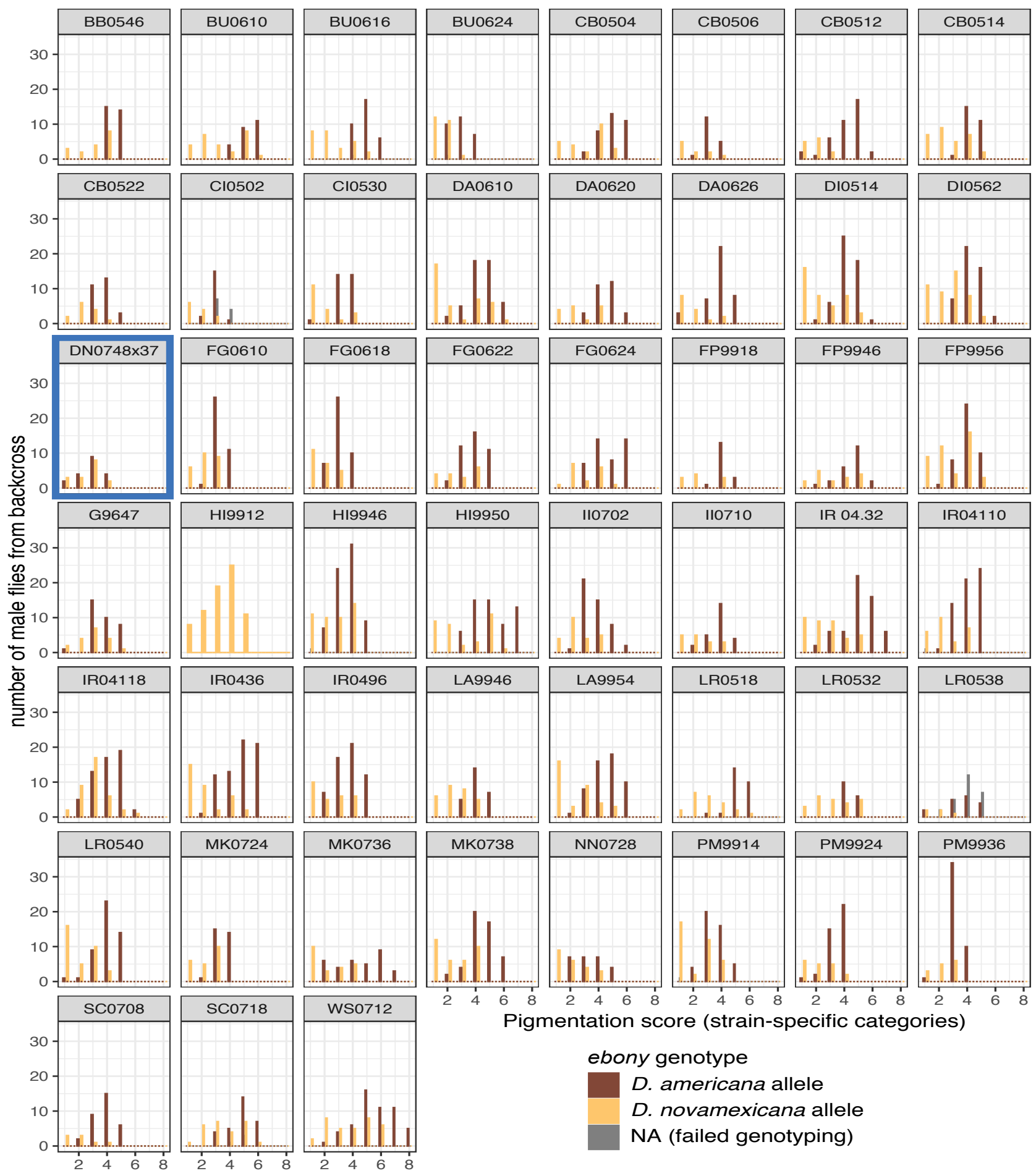

Supplementary Figure 4. Testing for function divergence of ebony. Distributions of pigmentation phenotypes for backcross progeny inheriting the $D$. americana (brown) or $D$. novamexicana allele (yellow) of the ebony gene from their $F_{1}$ hybrid mother are shown for each strain of $D$. americana tested, with the stain name shown at the top of each panel. Numbers of males in each pigmentation class are shown rather than proportions to communicate sample sizes. Grey bars indicate samples with failed genotyping reactions, which were most common for ebony with flies from strain LR0538. The genotyping assay seemed to fail to differentiate alleles in the backcross with strain HI9912. Dark blue box indicates no significant difference between $D$. americana and $D$. novamexicana alleles. 


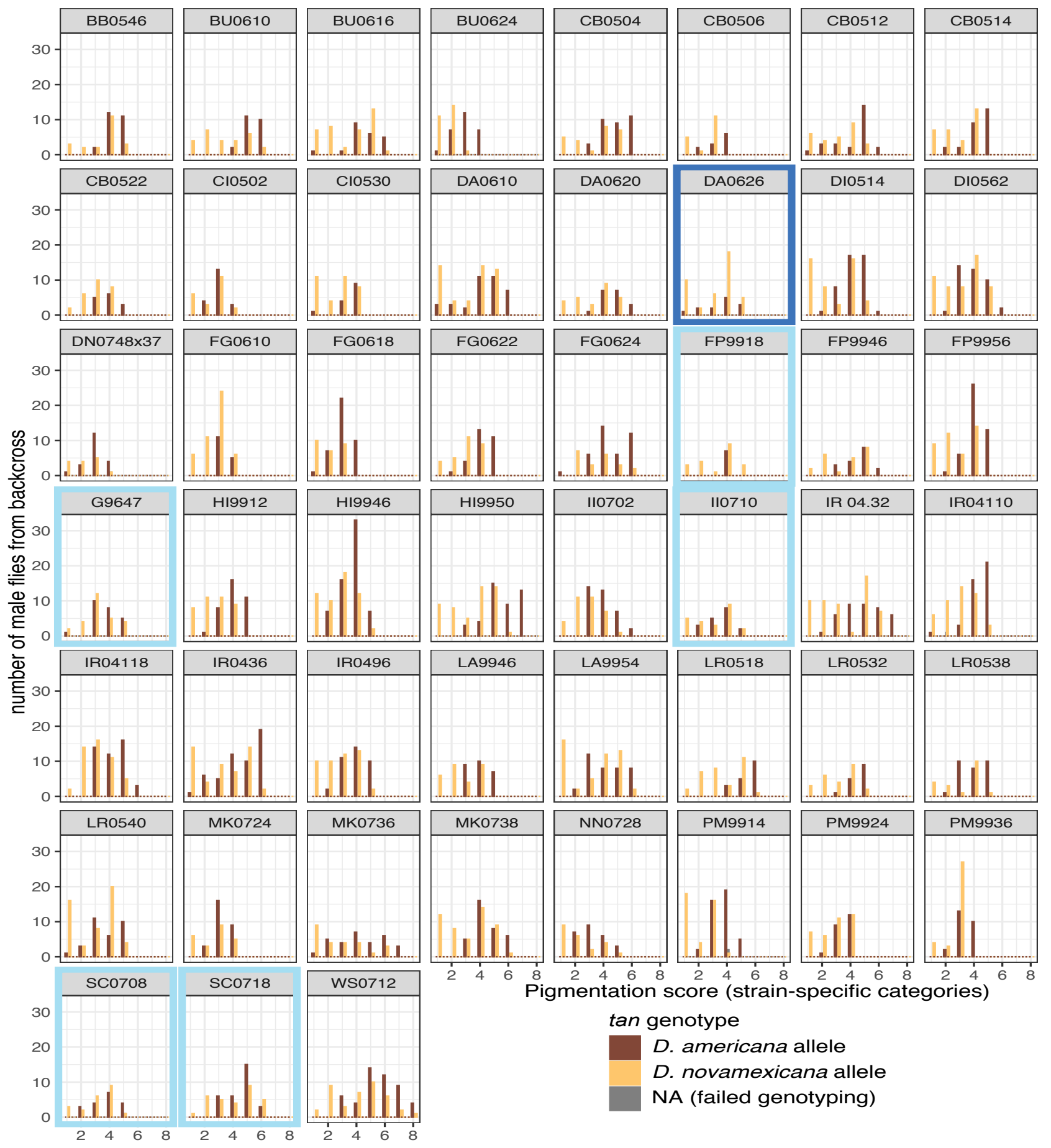

Supplementary Figure 5. Testing for function divergence of tan. Distributions of pigmentation phenotypes for backcross progeny inheriting the $D$. americana (brown) or $D$. novamexicana allele (yellow) of the tan gene from their $F_{1}$ hybrid mother are shown for each strain of $D$. americana tested, with the stain name shown at the top of each panel. Numbers of males in each pigmentation class are shown rather than proportions to communicate sample sizes. Grey bars indicate samples with failed genotyping reactions, of which very few were observed for ebony. Dark blue box indicates no significant difference between $D$. americana and $D$. novamexicana alleles. Light blue boxes indicate marginal evidence of equivalent alleles (P-values $=0.05$ or 0.06$)$. 


\section{Supplementary Tables}

Supplementary Table 1. Summary of strains used for sequence analysis and/or functional testing, including details of sites where their progenitors were collected.

Supplementary Table 2. Median pigmentation measure for each fly sampled from each strain of $D$. americana obtained using a custom-built fiber optic probe to measure light reflected off the fly's abdominal cuticle.

Supplementary Table 3. Standardization of pigmentation classes among all backcrosses.

Supplementary Table 4. yellow, tan, and ebony genotypes for male progeny of $\mathrm{F}_{1}$ hybrids backcrossed to $D$. novamexicana.

Supplementary Table 5. Results of permutation tests used to identify functional differences between $D$. americana and $D$. novamexicana alleles of yellow, tan, and ebony.

Supplementary Table 6. Genotypes of sites in tan used to test for an association with body pigmentation.

Supplementary Table 7. Genotypes of sites in ebony used to test for an association with body pigmentation.

Supplementary Table 8. Results from general linear models used to test for associations between body pigmentation and variable sites in tan and ebony.

\section{Supplementary Files}

Supplementary File 1. FASTA format summary of ebony allele sequences analyzed.

Supplementary File 2. FASTA format summary of tan allele sequences analyzed.

Supplementary File 3. Text file containing R code used for all analyses presented in the manuscript. 